\title{
Salience-Driven Value Construction for Adaptive Choice under Risk
}

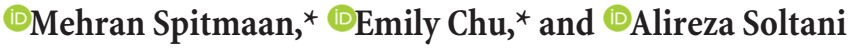 \\ Department of Psychological and Brain Sciences, Dartmouth College, Hanover, New Hampshire 03755
}

Decisions we face in real life are inherently risky and can result in one of many possible outcomes. However, most of what we know about choice under risk is based on studies that use options with only two possible outcomes (simple gambles), so it remains unclear how the brain constructs reward values for more complex risky options faced in real life. To address this question, we combined experimental and modeling approaches to examine choice between pairs of simple gambles and pairs of three-outcome gambles in male and female human subjects. We found that subjects evaluated individual outcomes of three-outcome gambles by multiplying functions of reward magnitude and probability. To construct the overall value of each gamble, however, most subjects differentially weighted possible outcomes based on either reward magnitude or probability. These results reveal a novel dissociation between how reward information is processed when evaluating complex gambles: valuation of each outcome is based on a combination of reward information whereas weighting of possible outcomes mainly relies on a single piece of reward information. We show that differential weighting of possible outcomes could enable subjects to make decisions more easily and quickly. Together, our findings reveal a plausible mechanism for how salience, in terms of possible reward magnitude or probability, can influence the construction of subjective values for complex gambles. They also point to separable neural mechanisms for how reward value controls choice and attention to allow for more adaptive decision making under risk.

Key words: attention; heuristics; prospect theory; reward; valuation

\section{Significance Statement}

Real-life decisions are inherently risky and can result in one of many possible outcomes, but how does the brain integrate information from all these outcomes to make decisions? To address this question, we examined choice between pairs of gambles with multiple outcomes using various computational models. We found that subjects evaluated individual outcomes by multiplying functions of reward magnitude and probability. To construct the overall value of each gamble, however, they differentially weighted possible outcomes based on either reward magnitude or probability. By doing so, they were able to make decisions more easily and quickly. Our findings illustrate how salience, in terms of possible reward magnitude or probability, can influence the construction of subjective values for more adaptive choice.

\section{Introduction}

Every decision we make entails some degree of risk and uncertainty and can result in one of many possible outcomes. For example, when choosing which restaurant to go to for lunch, one needs to consider many factors such as commute time, pricing, and wait time, each of which could vary depending on traffic, food availability, and the number of other customers who have

\footnotetext{
Received Sept. 29, 2018; revised April 17, 2019; accepted April 19, 2019.

Author contributions: M.S., E.C., and A.S. designed research; M.S., E.C., and A.S. performed research; M.S. and A.S. analyzed data; E.C. and A.S. wrote the paper.

This work was supported by a NSF Grant (EPSCoR Award 1632738) to A.S. We thank Daeyeol Lee for helpful comments on the paper.

The authors declare no competing financial interests.

*M.S. and E.C. contributed equally to this wok.

Correspondence should be addressed to Alireza Soltani at soltani@dartmouth.edu.

https://doi.org/10.1523/JNEUROSCI.2522-18.2019

Copyright $\odot 2019$ the authors
}

also chosen that restaurant. To compute the overall values for such complex options or to directly compare those options, the brain has to assign a value to each possible or relevant outcome based on reward information (e.g., expected reward and probability of a given outcome) followed by integration or direct comparison of those values. Any of these processes can be very daunting when there are multiple pieces of reward information and many possible outcomes. Therefore, to enable decisionmaking between real-world options, the brain must rely on certain mechanisms that simplify these valuation and choice processes to reduce mental effort and to make value-based decision making more adaptive (Payne et al., 1988).

Although prospect theory (Kahneman and Tversky, 1979), the standard model of choice under risk, has been very successful in capturing many aspects of choice (Wu and Gonzalez, 1996; Birnbaum and Navarrete, 1998; Gonzalez and Wu, 1999; Abdellaoui, 2000; Bruhin et al., 2010; Glöckner and Pachur, 2012), it 
fails to account for choice between gambles with more than two alternative outcomes (complex gambles). As a result, various models have been proposed to tackle valuation and choice between complex gambles, including cumulative prospect theory (Tversky and Kahneman, 1992), transfer of attention exchange (Birnbaum and Navarrete, 1998; Birnbaum, 2008), decision field theory (Busemeyer and Townsend, 1993), and salience theory of choice (Bordalo et al., 2012, 2013). Interestingly, most of these models use a "rank-dependent" strategy similar to what is proposed in cumulative prospect theory. This strategy assumes that possible outcomes are ranked based on different variables, such as reward probability, and the ranking, in turn, determines the influence of each outcome on the overall value via different (model-dependent) mechanisms. Despite the success of these models in capturing an overall pattern of choice between complex gambles, it is still unclear how mechanisms proposed in these models can be instantiated in the brain given the complicated computations required by such models.

Here, we used a combination of experimental and modeling approaches to reveal plausible neural mechanisms underlying valuation and choice between complex gambles. Considering its role in selection between multiple sources of information for further processing (Wolfe and Horowitz, 2004; Carrasco, 2011), we hypothesized that attention is also involved in the evaluation and choice between gambles with multiple outcomes. To test our hypothesis, we performed an experiment in which human subjects selected between pairs of gambles with only one non-zero outcome (simple gambles) and pairs of gambles with three nonzero outcomes (complex gambles). Furthermore, we developed a large family of models to capture the observed choice behavior. In these models, the value of a complex gamble was constructed by differentially weighting the value of its possible outcomes via a simple attentional mechanism. More specifically, attention could be guided by different types of reward information (e.g., reward magnitude, reward probability, expected value, etc.) to allow for differential weighting of possible outcomes. This essential feature of our model enabled evaluation of individual outcomes and their weighting to rely on different pieces of reward information (e.g., evaluation based on expected value but differential weighting based on magnitude). We fit subjects' choice behavior with our and competing models to assess our models' ability in capturing choice behavior and to address two key questions regarding the construction of reward value for complex gambles. First, how are individual outcomes of a complex gamble evaluated? Second, how are possible outcomes compared between two complex gambles, or equivalently, how are the values of possible outcomes combined to compute the overall value of a complex gamble?

\section{Materials and Methods}

Subjects. A total of 64 human subjects (38 females) were recruited from the Dartmouth College undergraduate student population. Subjects were compensated with money and/or "t-points", which were extracredit points for introductory classes in the department of Psychological and Brain Sciences at Dartmouth College. The base rate for compensation was $\$ 10 / \mathrm{h}$ or $1 \mathrm{t}$-point $/ \mathrm{h}$. All subjects were then additionally rewarded based on their performance, up to $\$ 15 / \mathrm{h}$. This additional performance-based compensation was always monetary. None of the subjects was excluded from our final data analyses. All experimental procedures were approved by the Dartmouth College Institutional Review Board, and informed consent was obtained from all subjects before participating in the experiment.

Experimental design. This study used a within-subject design. In two experimental sessions, each subject performed two tasks (simple-gamble and complex-gamble tasks) in which he/she selected between a pair of gambles on every trial and was provided with feedback about the outcome of the chosen gamble. In both tasks, gambles were presented as rectangular bars divided into different portions. A portion's color indicated the reward magnitude of that outcome, and its size signaled its probability (see Fig. $1 A-B$ ). This gamble presentation was adopted from a recent study in monkeys (Strait et al., 2014) because this design allowed us to accurately present complex gambles without using any numbers, making evaluation and decision making more intuitive. For both tasks, subjects were instructed to maximize their reward points, which later translated to monetary reward or t-points, by choosing the gamble that they believed was more likely to provide more reward points. The selected gamble was resolved following each choice according to probabilities associated with possible outcomes of the chosen gamble.

Before the beginning of each task, subjects completed a training session in which they selected between two sure gambles. These training sessions were used to familiarize subjects with the associations between four different colors (purple, magenta, green, and gray) and their corresponding reward values, which depended on the task. In both training sessions, all subjects selected the gamble with higher expected value (EV) on $>70 \%$ of the trials, indicating that they understood the color-reward associations. For the simple-gamble task, reward values were always 0,1 , 2 , and 4 points. For the complex-gamble task, 0 and 1 were used, but the other two reward values varied for each subject depending on their subjective utility (see Complex-Gamble Task). No reward (0 points) was always assigned to the gray color. The color-reward assignment remained consistent for each subject throughout both the training session and its corresponding task. The color-reward assignments, however, were randomized between subjects.

Simple-gamble task. The simple-gamble task consists of two types of trials: (1) choice between a sure option and a simple gamble with two outcomes of either a reward larger than that of the sure option or no reward with complementary probabilities, and (2) choice between two simple gambles (Fig. 1A). Reward magnitude and probability were represented by the color and length of the corresponding portion, respectively. Subjects evaluated a total of 63 unique gamble pairs, each of which was shown four times in a random order (total of 252 trials). To make this choice task nontrivial, the gamble pairs were constructed to be relatively similar in expected value.

Complex-gamble task. During the complex-gamble task, subjects selected between 70 unique pairs of gambles with three possible non-zero outcomes. Each gamble was presented four times in a random order (total of 280 trials). The complex-gamble task was similar to the simplegamble task with a few exceptions to increase the sensitivity of our experimental paradigm (Fig. 1B). More specifically, we constructed gambles with almost equal subjective values for each subject by tailoring the reward magnitudes and probabilities of individual gamble outcomes to each subject. First, the middle and large reward values were tailored for each subject according to their utility function estimated from their choice behavior in the simple-gamble task. No reward ( 0 points) and the small reward (1 point) remained unchanged from the simple-gamble task, whereas the middle and large magnitudes were adjusted to have approximately double and quadruple the small reward's utility, respectively. We kept the maximum value of reward magnitude at 10 , resulting in the medians of 3 and 8 for the middle and large rewards, respectively. Although reward magnitudes associated with each color might differ between the simple-gamble and complex-gamble tasks for individual subjects, the relative order of color-reward association did not. For example, the largest reward value in the complex-gamble task would be associated with the color previously corresponding to 4 points in the simple-gamble task, etc.

To construct pairs of three-outcome gambles that are very close in subjective utility for each subject, we also adjusted probabilities of alternative outcomes based on the subject's estimated utility and probability weighting functions from the simple-gamble task. More specifically, the combination of outcome probabilities for each gamble was selected from one of the following sets: $\{0.6,0.2,0.2\},\{0.4,0.3,0.3\},\{0.5,0.3,0.2\}$, and $\{0.4,0.4,0.2\}$. From the set of all possible gambles, we then picked pairs of gambles for which the difference in the subjective values was less than the 
A

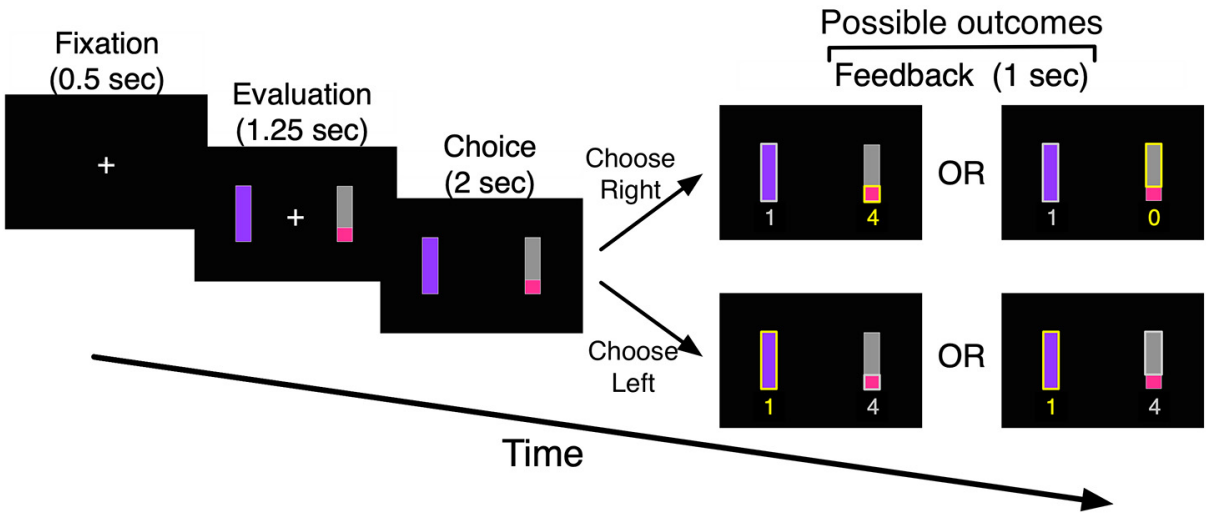

B

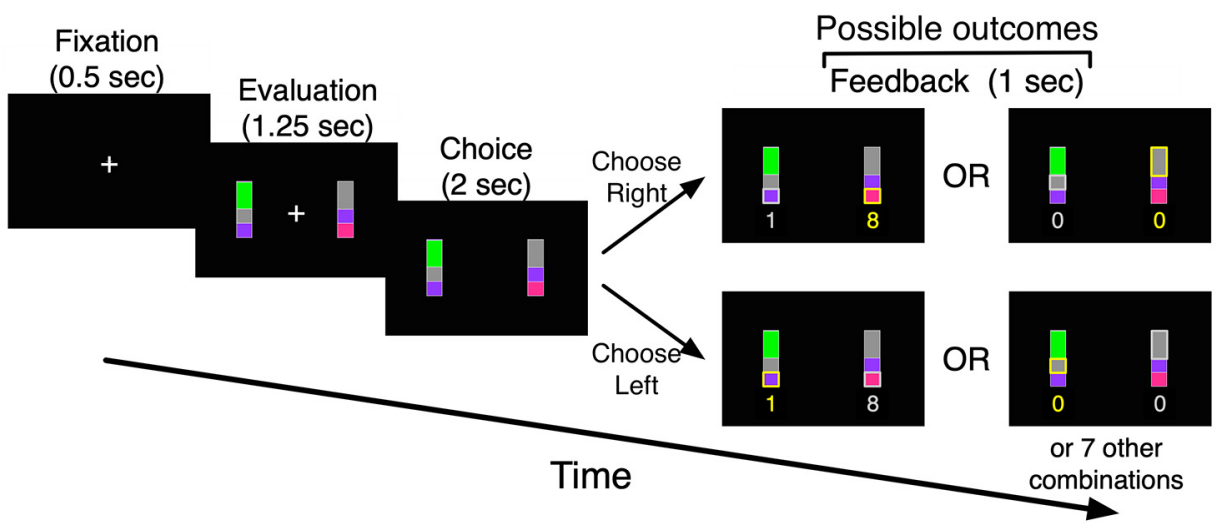

C

\section{Quantities used for evaluation}

$m$

$u(m)$

$p$

$w(p)$

EV

$E U$

EV with w(p)

SU

D

Weighting strategy (parameters)

\begin{tabular}{|c|c|c|c|c|c|}
\hline \multicolumn{2}{|c|}{ strategy $1:(\sigma)$} & \multicolumn{2}{|c|}{ strategy 2: $(\sigma)$} & \multicolumn{2}{|c|}{ strategy $3:\left(\sigma, \beta_{b}\right)$} \\
\hline $1 / 3\{$ & $V_{b}$ & $1 / 2\{$ & $v_{b}$ & $\beta_{b}\{$ & $v_{b}$ \\
\hline $1 / 3\{$ & $\mathrm{V}_{\mathrm{m}}$ & $1 / 2\{$ & $V_{m}$ & $\left(1-\beta_{b}\right)\{$ & $\mathrm{V}_{\mathrm{m}}$ \\
\hline $1 / 3\{$ & $v_{w}$ & & $\mathrm{~V}_{\mathrm{w}}$ & & $\mathrm{v}_{\mathrm{w}}$ \\
\hline
\end{tabular}

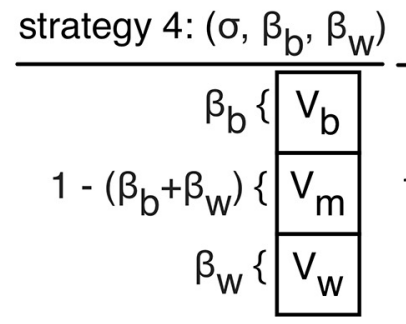

strategy 5: $\left(\sigma, \beta_{b}, \beta_{w}, \beta_{b}^{\prime}\right)$

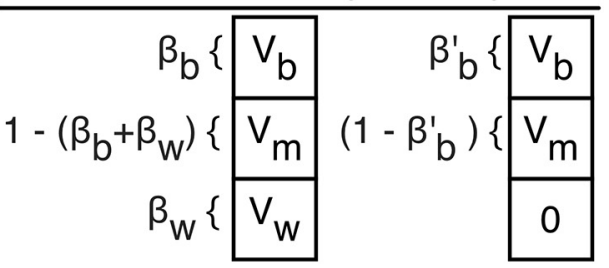

Figure 1. Experimental paradigm and alternative strategies for the construction of reward value. On each trial of the simple-gamble task $(\boldsymbol{A})$ subjects selected between either a sure option and a simple gamble or a pair of simple gambles before receiving feedback. In the complex-gamble task $(\boldsymbol{B})$ subjects selected between pairs of complex gambles. A simple gamble could yield reward or no reward with complementary probabilities, whereas a complex gamble offered three possible outcomes. To model the construction of reward value, we considered eight alternative quantities to evaluate individual gamble outcomes $(\boldsymbol{C} ; m$, magnitude; $u(m)$, utility function; $p$, probability; $w(p)$, probability weighting function; $\mathrm{EV} ; \mathrm{EV}$ with $w(p)$; $\mathrm{EU} ; \mathrm{SU})$. We considered six of these quantities $\left(m, p, \mathrm{EV}, \mathrm{EV}\right.$ with $w(p), \mathrm{EU}$, and SU) to sort possible outcomes as the best, middle, and worst $\left(V_{\mathrm{b}}, V_{\mathrm{m}}\right.$, and $V_{\mathrm{w}}$ correspond to the values of best, middle, and worst outcomes, respectively). The quantities $u(m)$ and $w(p)$ were not considered for sorting because they produce the same sorting results as $m$ and $p$, respectively. We also considered five different weighting strategies by which the values of individual outcomes can be combined to form the overall gamble value $(\boldsymbol{D})$. The parameter $\sigma$ represents the stochasticity in choice, which transforms the difference in reward values to the probability of choice. $\beta_{\mathrm{b}}$ and $\beta_{w}$ are fixed model parameters denoting the differential weights associated with the best and worst outcomes, respectively. Meanwhile, $\beta^{\prime}{ }_{\mathrm{b}}$ denotes a separate weight associated with the best outcome when the gamble includes a zero-magnitude outcome.

$5 \%$ of the difference between the maximum and minimum subjective values of all possible pairs. We required the probabilities of the three outcomes to differ from one another by a value larger than or equal to 0.1 to ensure that differences in probabilities were easily visually discernable.
Our procedure and the large set of possible pairs guaranteed that there was no correlation between reward magnitude and probability of each gamble outcome (Pearson correlation: $r=0.01, p=0.78$; Spearman correlation, $r=0.026, p=0.33$ ). Finally, we also included 20 "catch" 
trials in which one of the gambles was better than the other with respect to both reward magnitude and probability. Catch trials were included to determine whether subjects were attentive to the presented gambles or not.

Modeling valuation and choice between simple gambles. We fit choice behavior of individual subjects during the simple-gamble task using four quantities to assign value to the only non-zero gamble outcome. These include: expected value (EV $=m \times p)$; expected value with the probability weighting function [EV with $w(p)=m \times w(p)$ ]; expected utility $[\mathrm{EU}=u(m) \times p)$; and subjective utility $(\mathrm{SU}=u(m) \times w(p)]$. We considered a power law for the utility function:

$$
u(m)=m^{\rho}
$$

where $\rho>0$ measures the curvature of the utility function. The probability weighting function (subjective probability) was modeled using the one-parameter Prelec function (Prelec, 1998):

$$
w(p)=\exp \left(-(-\ln p)^{\gamma}\right)
$$

where $\gamma>0$ measures the distortion of the probability weighting function. Finally, using reward values assigned to the two gambles on each trial, the probability that the subject would choose the gamble on the right $\left(P_{\mathrm{R}}\right)$ was computed as follows:

$$
P_{R}=\frac{1}{1+\exp \left(-\left(V_{R}-V_{L}\right) / \sigma\right)},
$$

where $V_{R}$ and $V_{L}$ denote the value of the right and left gambles, respectively, and $\sigma$ is a model parameter that measures stochasticity in choice by transforming the difference in reward values to the probability of choice.

Modeling valuation and choice between complex gambles. We used a large family of models to fit choice behavior during the complex-gamble task. Our models differed in their assumptions about how individual outcomes of a given gamble are evaluated ( 8 possible quantities also referred to as strategies; Fig. 1C), how individual outcome values are sorted ( 6 possible quantities also referred to as strategies), and how the sorted outcomes are combined to form the overall value of a threeoutcome gamble ( 5 possible strategies; Fig. $1 D$ ). Reward values assigned to the two gambles on each trial were used to determine the probability of selecting between the two gambles based on Equation 3. More formally, the value of an option $X, V_{X}$, depends on sorting, evaluation, and weighting strategies as follows:

$$
V_{X}=\sum_{k=1}^{3} V_{<\text {eval }>, X}^{k} \times D W_{<\text {stratgey\#> }}^{I_{<\text {sort }, X}^{k}}
$$

where $V_{<\text {eval }>, X}^{k}$ is the value of outcome $k$ of option $X$, $D W_{<<\text {starteg } \#>X X}^{I_{k}^{k} \text { sor }>X}$ is the weight of outcome $k$ of option $X$ based on sorting index $I_{<\text {sort }>, X}^{k}$ and the weighting strategy. $V_{<\text {eval }>, X}^{k}$ can be equal to magnitude $(m)$, utility $[u(m)]$, probability $(p)$, or weighted probability $[w(p)]$, which we collectively refer to "single-attribute" evaluation strategies. $V_{<\text {eval }>, X}^{k}$ can also be equal to expected value $(m \times p)$, expected utility $[u(m) \times(p)]$, expected value with weighted probability $[m \times$ $w(p)]$, or subjective utility $[u(m) \times w(p)]$, which we collectively refer to as "combined-attribute" evaluation strategies. Sorting index for each outcome, $I_{<\text {sort }>, X}^{k} \in\{b:$ best, $m:$ middle, $w:$ worst $\}$, is computed after sorting outcomes based on a given sorting quantity:

$I_{<\text {sort }>, X}^{k}=\operatorname{sort}\left(V_{<\text {eval }>, X}^{k}\right.$, sort $\left._{\text {qunatity }}\right)$,

$\operatorname{sort}_{\text {qunatity }}=\{m, p, m \times p, u(m) \times p, m \times w(p), u(m) \times w(p)\}$.

Note that sorting outcomes based on $m$ and $u(m)$, and for $p$ and $w(p)$ would be identical, reducing the number of sorting strategies to six.

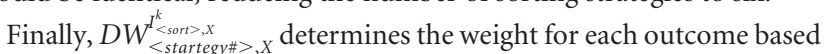
on the sorting index and one of five possible weighting strategies (Fig.
$1 D)$. Weighting Strategy 1 equally weights the three possible outcomes, $D W=\left[\beta_{b}=\frac{1}{3}, \beta_{m}=\frac{1}{3}, \beta_{w}=\frac{1}{3}\right]$, where $\beta_{b}, \beta_{m}$, and $\beta_{w}$ denote the weight assigned to the best, middle, and worst outcome, respectively. Strategy 2 assigns equal weights to the best and middle outcomes and ignores the worst outcome, $D W=\left[\beta_{b}=\frac{1}{2}, \beta_{m}=\frac{1}{2}, \beta_{w}=0\right]$. Strategy 3 uses a single parameter to distribute weights between the best and middle outcomes while assigning zero weight to the worst outcome, $D W=\left[\beta_{b},\left(1-\beta_{b}\right), 0\right]$. Strategy 4 freely distributes weights to the three outcomes using two parameters, $D W=\left[\beta_{b}, 1-\left(\beta_{b}+\beta_{w}\right), \beta_{w}\right]$. Strategy 5 is similar to Strategy 4 but uses a separate parameter for distributing weight when there is a zero-magnitude outcome. We also tested an additional strategy with equal weighting for middle and worst outcomes. The results for this strategy are not reported because this strategy was not able to successfully capture the choice data of any subject. Finally, we note that although weighting Strategies 1,2, and 3 can be considered as special cases of Strategy 4, we tested these strategies since they have fewer parameters, which could result in a better goodness-of-fit when the number of parameters were considered (see Model comparison and data analysis). All the possible combinations of strategies resulted in the generation of 240 alternative models ( 8 possible strategies for evaluating individual outcomes, 6 for sorting outcomes, and 5 for weighting).

Competing models of valuation and choice between complex gambles. To compare our model with existing models of valuation and choice between complex gambles, we fit choice behavior of our subjects during the complex-gamble task using four different rank-dependent models. This includes cumulative prospect theory (CPT; Tversky and Kahneman, 1992), transfer of attention exchange (TAX; Birnbaum and Navarrete, 1998; Birnbaum, 2008), salience theory of choice (STC; Bordalo et al., 2012, 2013), and decision field theory (DFT; Busemeyer and Townsend, 1993). In the following sections, we provide a summary of these models and how they are implemented.

Cumulative prospect theory. CPT generalizes prospect theory for choice under uncertainty by extending PT in multiple ways. Importantly, by adopting a cumulative representation for probability, CPT can be applied to gambles with more than two non-zero outcomes and removes the need for the editing rules of combination and dominance detection. More specifically, for gambles with strictly non-negative outcomes $\left(m_{1} \geq m_{2} \geq \ldots \geq m_{n} \geq 0\right)$, the utility of gamble $G, C P T(G)$, is equal to:

$$
\operatorname{CPT}(G)=\sum_{i=1}^{n}\left[W\left(\sum_{j=1}^{i} p_{j}\right)-W\left(\sum_{j=1}^{i-1} p_{j}\right)\right] u\left(m_{i}\right)
$$

where $W\left(P_{i}\right)$ is the cumulative weighting function of probability of receiving more than $m_{i}\left(P_{i}=\sum_{j=1} p_{j}\right)$, with boundary conditions of $W(0)=0$ and $W(1)=1$. We used the following form of the cumulative weighting function:

$$
W(P)=\frac{P^{\gamma}}{\left[P^{\gamma}+(1-P)^{\gamma}\right]^{1 / \gamma}},
$$

where $\gamma$ is a free parameter. Values of $\gamma>1$ and $\gamma<1$ correspond to $\mathrm{S}$-shaped and inverse-S-shaped curves, respectively.

Transfer of attention exchange. In TAX, the utility of a gamble is equal to a weighted average of the utilities of the outcomes (Birnbaum and Navarrete, 1998; Birnbaum, 2008). These weights, referred to as "configural" weights, depend on the probability and rank of the outcome branches and therefore, the relationship between branches. This model assumes that a decision-maker deliberates by attending to possible outcomes of an action depending on their risk attitude. Not only can branches leading to larger rewards attract more attention but branches leading to lower-value outcomes can also attract greater attention if a person is risk-averse. Importantly, the weights of branches result from transfers of attention from one branch to another. If there were no configural effects, then each branch would have weights purely as a function of cumulative outcome probability, $W(P)$. However, depending on the subject's point of view (i.e., risk attitude), weight is transferred from 
branch to branch. For example, for a risk-averse subject, weight can be transferred from a higher-value branch $k$ to a lower-value branch $i$ $\left(m_{k} \geq m_{i}\right)$. If $\omega\left(p_{i}, p_{k}, n\right)$ represents the weight transferred from branch $k$ to branch $i$, the value of gamble $G$ in TAX can then be written as follows:

$$
\operatorname{TAX}(G)=\frac{\sum_{i=1}^{n} t\left(p_{i}\right) u\left(m_{i}\right)+\sum_{i=2}^{n} \sum_{k=1}^{i-1}\left[u\left(m_{i}\right)-u\left(m_{k}\right)\right] \omega\left(p_{i}, p_{k}, n\right)}{\sum_{i=1}^{n} t\left(p_{i}\right)},
$$

where $t($.$) is a monotonic function of probability and \omega($.$) is equal to:$

$$
\omega\left(p_{i}, p_{k}, n\right)=\left\{\begin{array}{l}
\frac{\delta \times t\left(p_{k}\right)}{n+1} \delta>0 \\
\frac{\delta \times t\left(p_{i}\right)}{n+1} \delta \leq 0
\end{array},-1<\delta<1 .\right.
$$

Indicating that the weight transferred is a fixed proportion of the weight of the branch giving up the weight. This formulation represent a general case, but assuming lower-value branches receive greater weights $(\delta>0)$, a special TAX model (Birnbaum, 2008) can be written for the value of three-outcomes gambles, $G=\left(m_{1}, p_{1} ; m_{2}, p_{2} ; m_{3}, p_{3}\right)$, where $m_{1} \geq m_{2} \geq$ $m_{3} \geq 0$, as follows:

$$
\operatorname{TAX}(G)=\frac{A u\left(m_{1}\right)+B u\left(m_{2}\right)+C u\left(m_{3}\right)}{A+B+C},
$$

where

$$
\begin{aligned}
& A=w\left(p_{1}\right)-2 \delta w\left(p_{1}\right) / 4 \\
& B=w\left(p_{2}\right)-\delta \times w\left(p_{2}\right) / 4+\delta \times w\left(p_{1}\right) / 4, \\
& C=w\left(p_{3}\right)+\delta \times w\left(p_{1}\right) / 4+\delta \times w\left(p_{2}\right) / 4
\end{aligned},
$$

where $w($.$) is the probability weighting function (Eq. 2).$

Salience theory of choice. In STC, the decision-maker's attention is drawn to (precisely defined) salient payoffs (Bordalo et al., 2012, 2013). This leads the decision-maker to a context-dependent representation of gambles in which true probabilities are replaced by decision weights distorted in favor of salient payoffs. By specifying decision weights as a function of payoffs, STC provides a unified account of many empirical phenomena, including frequent risk-seeking behavior, invariance failures such as the Allais paradox, and preference reversals. The value of a gamble in STC is computed by weighting possible outcomes based on their salience as follows [for gambles with three outcomes, $G=$ $\left.\left(m_{1}, p_{1} ; m_{2}, p_{2} ; m_{3}, p_{3}\right)\right]$ :

$$
\operatorname{STC}\left(G_{l}\right)=\sum_{i=1}^{3} W\left(p_{i}^{l}\right) \omega_{i}^{l} u\left(m_{i}^{l}\right)
$$

where $W\left(\right.$.) is the cumulative weighting function (Eq. 7) and $\omega_{i}^{l}$ is "salient weight" for outcome $i$ of gamble $l$. The salient weight for each outcome is computed using the salient ranking of each outcome:

$$
\omega_{i}^{l}=\frac{\delta^{r_{i}}}{\left(\sum_{t} \delta^{r_{t}} \times p_{t}^{l}\right)},
$$

where $\delta \in(0,1]$ is a free parameter and $r_{i}^{l} \in\{1, \ldots,|I|\}$ is the salient ranking of outcome $i$ in gamble $G_{l}$ (lower $r_{i}^{l}$ indicates higher salience). Given two outcomes of gamble $G_{l}, i$ and $\tilde{i} \in I$, outcome $i$ is considered to be more salient than outcome $\tilde{\imath}$ if $\sigma\left(m_{i}^{l}, m_{i}^{k}\right)>\sigma\left(m_{i}^{l}, m_{\tilde{\imath}}^{k}\right)$, where $\sigma\left(m_{i}^{l}, m_{i}^{k}\right)$ is the function measuring the saliency based on the outcome magnitude in the two competing gambles $l$ and $k$ :

$$
\sigma\left(m_{i}^{l}, m_{i}^{k}\right)=\frac{\left|m_{i}^{l}-m_{i}^{k}\right|}{\left|m_{i}^{l}\right|+\left|m_{i}^{k}\right|+\theta},
$$

and $\theta>0$ is a free parameter.

Decision field theory. Busemeyer and Townsend (1993) derive DFT from an intuitive but sophisticated computational logic. Suppose that a decision maker attempts to choose according to rank-dependent values of alternative gambles (such as those given by CPT) but does not have an algorithm for effortlessly and quickly multiplying utilities and weights together. The decision-maker could instead proceed by sampling the possible utilities of options in proportion to their decision weights, computing the running sums of these sampled utilities for each option, and stopping (and choose) when the difference between the sums exceeds some threshold determined by the cost of sampling. Considering this algorithm, the probability of choosing the right option based on the difference in values of the right and left options can be simplified as follows:

$$
P_{R}=F\left(\frac{V_{R}-V_{L}}{D\left(V_{R}, V_{L}\right)}, \sigma\right),
$$

where $F$ is the sigmoid function $[F(x, \sigma)=1 /(1+\exp (-x / \sigma))]$, and

$$
V_{X}=\sum_{i=1}^{3} W\left(p_{X i}\right) u\left(m_{X i}\right)
$$

$D\left(V_{R}, V_{L}\right)=\sqrt{\operatorname{Var}\left(V_{R}-V_{L}\right)}$

$$
=\sqrt{\operatorname{Var}\left(V_{R}\right)+\operatorname{Var}\left(V_{L}\right)-2 \operatorname{Cov}\left(V_{R}, V_{L}\right)}
$$

$$
\operatorname{Var}\left(V_{X}\right)=\sum_{i=1}^{3} W\left(p_{X i}\right)\left[u\left(m_{X i}\right)-V_{X}\right]^{2}
$$

$\operatorname{Cov}\left(V_{R}, V_{L}\right)=\left(\frac{1}{3}\right) \sum_{i=1}^{3}\left(\left(W\left(p_{R i}\right)\left[u\left(m_{R i}\right)-V_{R}\right]\right)\right.$

$$
\left.\times\left(W\left(p_{L i}\right)\left[u\left(m_{L i}\right)-V_{L}\right]\right)\right)
$$

$W($.$) is the cumulative weighting function (Eq. 7).$

Model comparison and data analysis. We fit choice data using our models and the aforementioned competing models by minimizing the negative log likelihood of the predicted choice probability given different model parameters. Minimization was done using the fminsearch function in MATLAB (MathWorks) over 50 initial model parameters. For model comparison and selection, we used the Akaike information criterion (AIC) or the Bayesian information criterion (BIC) to account for the different number of parameters in different models. Although the minimum AIC (and BIC) values varied across subjects, reflecting differences in how each subject's choice behavior could be best captured, all model comparisons were performed using AIC or BIC values within each subject, making our statistical tests more robust. We also examined the quality of model selection using Vuong's test (Vuong, 1989; see below). We note that because of the small number of trials (280) and the large number of gamble pairs (70), it is impossible to perform cross validation on data from individual subjects. Additionally, cross validation across subjects is futile because of individual variability and differences in risk attitude.

The fitting of choice behavior in the simple-gamble task allowed us to estimate the utility and probability weighting function for individual subjects. To identify the strategies used for sorting, evaluating, and weighting in the complex-gamble task, we computed and compared the goodness-of-fit (AIC and BIC) across all models to find the best overall model. The best model was then used to determine the strategies used for sorting, evaluating, and weighting. In other words, we did not find the best average model across each strategy dimension and instead, found the best overall model across all strategy dimensions.

To further examine the quality of model comparison, we also used Vuong's test (Vuong, 1989) for model selection. Specifically, Vuong's test determines the best model as the model with the log likelihood (LL) significantly smaller than the one of the second best model. Considering $N$ samples of LL values for each model, Vuong statistic for comparing models $i$ and $j$ is calculated using the following equation: 


$$
\begin{gathered}
\text { Vuong statistic }=\frac{\left[\operatorname{LLR}\left(\text { model }_{i}, \text { model }_{j}\right)-C_{i j}\right]}{\sqrt{N \times V}} \sim N(0,1) \\
L L R\left(\text { model }_{i}, \text { model }_{j}\right)=\sum_{k=1: N} L L_{i, k}-L L_{j, k}, \\
C_{i j}=\frac{K_{i}-K_{j}}{2} \log N \\
V=\operatorname{var}\left(L L_{i, k}-L L_{j, k}\right)_{k=1: N}
\end{gathered}
$$

where $L L R$ is the sum of LL ratios for the two models, $C_{i j}$ is a correction term for the difference of degrees of freedom between two models, $V$ is the variance of LL ratios between two models, and $K_{i}$ and $K_{j}$ are the numbers of parameters in models $i$ and $j$, respectively. It has been shown that Vuong statistic follows a standard normal distribution $N(0,1)$. As a result, model $i$ can be considered better than $j$ if Vuong statistic $>1.96$ and vice versa.

Finally, to quantify how easily a subject can distinguish between a pair of gambles based on their subjective values, we defined "discriminability" as follows:

$$
\text { Discriminability }=\sum P_{R}\left(\text { if } V_{R} \geq V_{L}\right)+\left(1-P_{R}\right)\left(\text { if } V_{R}<V_{L}\right),
$$

where $V_{\mathrm{R}}$ and $V_{\mathrm{L}}$ indicate the value of the left and right gambles, respectively, $P_{\mathrm{R}}$ is the probability of selecting the right gamble, and the sum is computed over all unique pairs of gambles. The chance level of discriminability is equal to 0.5 .

Statistical analysis. We fit choice data using different models to find the best model (using AIC and/or BIC) and estimating model parameters, and moreover, used Vuong's test to ensure the quality of model comparison (for more details, see Model comparison and data analysis). Model parameters were compared using two-sided sign test. We used Pearson's $\chi^{2}$ test of homogeneity to compare ratio of subjects that adopted different types of strategies. For correlation analyses, we used both Pearson and Spearman correlations. For all tests, $p<0.05$ was considered statistically significant. We compared discriminability and changes in subjective values due to differential weighting within individual subjects. We also had a between-subject comparison for the measure of discriminability between simple- and complex-gamble tasks.

Model recovery. To test the ability of our fitting procedure in capturing the proposed mechanisms for the construction of overall reward value and extracting the correct parameters, we simulated choice data using the proposed models over a wide range of model parameters for the same complex-gamble task performed by the subjects. We then fit the simulated data with each of these models to compute the goodness-of-fit (in terms of AIC) and estimate the original model parameters used to simulate the data. Given the large number of possible models (240), we only considered the most frequent models identified in the experimental data for this analysis. More specifically, we only considered three parameters for sorting the values of individual outcomes ( $m, p$, and EV) because sorting based on the other five quantities $[u(m), w(p), \mathrm{EU}, \mathrm{EV}$ with $w(p)$, and $\mathrm{SU}]$ resulted in very similar outcomes as the former quantities. Moreover, we only considered Strategy 4 for weighting of values since this was the most frequently used strategy for differential weighting. Based on these specifications, we narrowed our analysis to a total of 24 models. For each of these 24 models, we generated choice data for the same complex-gamble task performed by the subjects (with the same number of parameters, trials, etc.) and fit the various choice data using each of the 24 models.

For simulations presented in Figure 3, we generated choice data using different combinations of strategies for sorting and evaluating, and weighting strategies with different sets of parameters. More specifically, we used different combinations of $\beta_{b}, \beta_{m}$, and $\beta_{w}$ values: $\beta_{b}=\frac{1}{3}, \beta_{m}=\frac{1}{3}$, and $\beta_{w}=\frac{1}{3}$, corresponding to weighting Strategy $1 ; \beta_{b}=\frac{1}{2}, \beta_{m}=\frac{1}{2}$, and $\beta_{w}=0$, corresponding to Strategy $2 ; \beta_{b}=[0: 0.25: 1], \beta_{m}=1-\beta_{b}$, and $\beta_{w}=0$, corresponding to Strategy 3; and finally $\beta_{b}=[0]$ and $\beta_{w}=[0.25: 0.25: 1], \beta_{b}=[0.25]$ and $\beta_{w}=[0.25: 0.25: 0.75], \beta_{b}=[0.5]$ and $\beta_{w}=[0.25: 0.25: 0.5]$, and $\beta_{b}=[0.75]$ and [for all of which $\left.\beta_{m}=1-\left(\beta_{b}+\beta_{w}\right)\right]$, corresponding to Strategy 4 . Nonetheless, our method can correctly identify the weighting strategy, as evident in Figure 5 that shows only a small bias in estimation of $\beta$ values for the most comprehensive weighting strategy (Strategy 4). For all these simulations, we used the median values of the estimated parameters from our subjects' probability weighting and utility functions ( $\rho=0.63$ and $\gamma=0.88$ ).

\section{Results}

The experiment consisted of two tasks. In the first task, subjects selected between either a sure gamble and a simple gamble, or between a pair of simple gambles (simple-gamble task; Fig. 1A). In the second task, decisions were made between pairs of threeoutcome gambles (complex-gamble task; Fig. $1 B$ ). In both tasks, gambles were presented as rectangular bars divided into different portions. A portion's color indicated the reward magnitude of that outcome, and its size signaled its probability (see Materials and Methods).

To examine whether subjects comprehended the objective of both tasks, we computed the probability of selecting the gamble with a larger EV on a given trial for each subject. During the simple-gamble task, subjects selected the gamble with a higher EV more often than chance $(0.5)$, with a median equal to 0.79 across all subjects (two-sided sign test, $p=1.12 \times 10^{-16} ; d=4.11$ ). This tendency was weaker in the complex-gamble task, with the median equal to 0.57 (two-sided sign test, $p=6.17 \times 10^{-4} ; d=$ 0.68 ). Less frequent selection of gambles with a higher $E V$ (i.e., more noisy behavior) was expected during the complex-gamble task since the pairs of presented gambles were closer in EV. However, on catch trials of the complex-gamble task (where one of the gambles was better than the other with respect to both reward magnitude and probability), subjects selected the better option more often than chance, with a probability larger than 0.7 and a median of 0.95 (two-sided sign test, $p=1.08 \times 10-19 ; d=4.67$ ). Together, these results illustrate that subjects understood the objective of both tasks and were motivated earn reward points.

\section{Evaluation of simple gambles conformed to PT}

We used various models to fit choice behavior to identify how individual subjects constructed the overall value of gambles in each task (see Materials and Methods). For the simple-gamble task, we assumed that subjects used one of the following four quantities to evaluate the only non-zero outcome of each gamble: EV; EV with the probability weighting function $[w(p)]$; EU; and SU.

Fitting of individual subjects' choice behavior showed that the model based on SU provided the best fit for the majority ( $83 \%$ ) of subjects in the simple-gamble task (Fig. $2 A$ ). This indicates that subjects mainly used subjective utility to evaluate simple gambles. In addition, the estimated utility and probability weighting functions conformed to the predictions of PT. More specifically, the majority of subjects ( 56 of 64 , corresponding to $\sim 88 \%$ of subjects) exhibited concave utility functions (Fig. $2 B$ ), and most subjects ( 43 of 64 , corresponding to $\sim 67 \%$ of subjects) exhibited an inverse-S-shaped probability weighting function (Fig. 2C). Overall, these results demonstrate that PT can successfully account for choice between simple gambles.

\section{Modeling evaluation and choice between complex gambles}

We hypothesized that attention is involved in the evaluation and choice between gambles with multiple outcomes. Therefore, we developed a family of models that rely on the assumption that the values of individual gamble outcomes are differentially weighted 
A

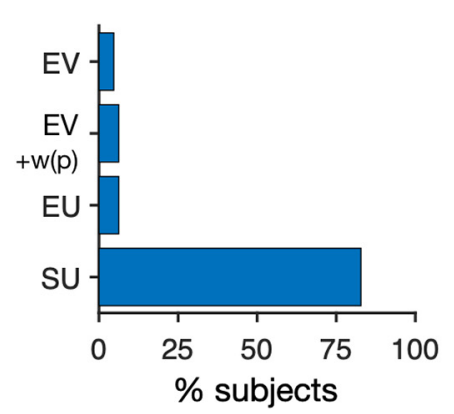

B

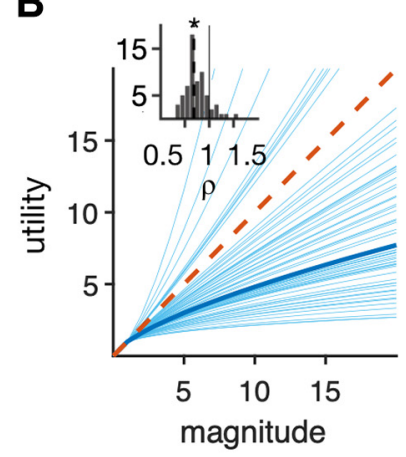

C

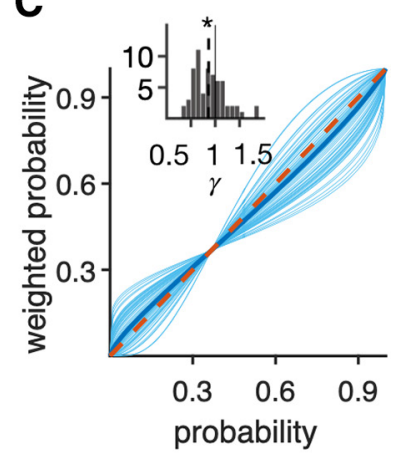

Figure 2. Prospect theory captured choice between simple gambles in most subjects. $A$, Plot shows the fraction of subjects for whom a given model provided the best fit. The model based on SU provided the best fit for the majority ( 53 of 64 ) of subjects. $\boldsymbol{B}$, Plotted is the estimated utility function for individual subjects (light blue curves) based on the model with SU. The dark blue curve shows a utility function formed based on the median of the distribution of estimated exponents of the utility function $(\rho)$, as shown in the inset. The red dashed line shows the unity line. The solid and dashed lines in the inset indicate $\rho=1$ and the median of $\rho$ values ( $0.38<\rho<1.53$, median: $\rho=0.63$ ), respectively; the asterisk shows the median is significantly different from 1 (tw0-sided sign test, $p=2.01 \times 10^{-34}, d=3.10$ ). Overall, most subjects exhibited a concave utility function. $C$, Plotted is the estimated probability weighting function for individual subjects. The dark blue curve shows a probability weighting function formed based on the median of the distribution of estimated exponents of the probability weighting function ( $\gamma$ ), as shown in the inset. The solid and dashed lines in the inset indicate $\gamma=1$ and the median of $\gamma(0.36<\gamma<1.89$, median: $\gamma=0.88)$, respectively. The asterisk shows the median is significantly different from 1 (two-sided sign test, $p=0.008, d=2.70)$. Overall, most subjects exhibited an inverse-S-shape probability weighting function as predicted by PT.

A

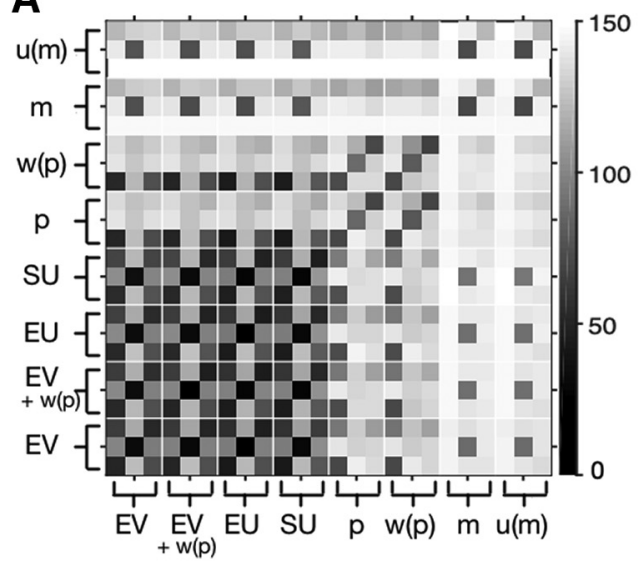

B

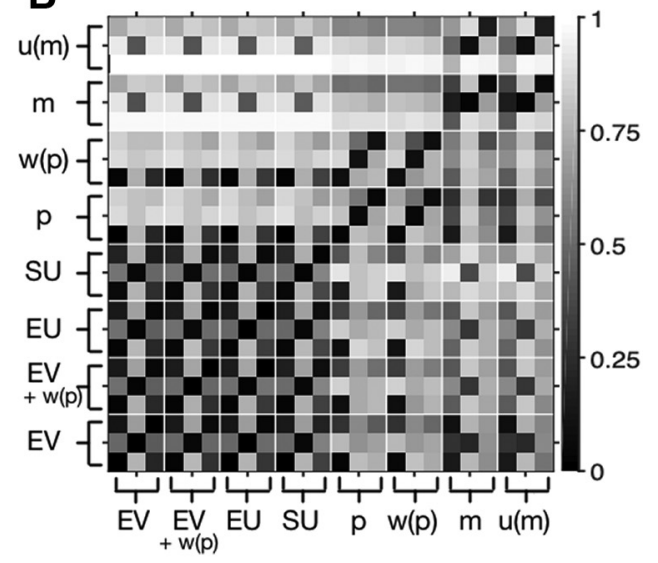

Figure 3. Fitting method was able to correctly identify the quantity used for evaluating individual gamble outcomes and for sorting in most cases. $\boldsymbol{A}$, Plot shows the goodness-of-fit (in terms of the average AIC over a set parameters) for fitting choice data generated with a given model and fit with one of the possible 24 models. The models used to generate and fit the data are indicated on the $x$ - and $y$-axes, respectively. For each quantity used for evaluating individual outcomes (shown on the axes), there are three ways to sort based on magnitude, probability, and EV (first, second, and third elements; not shown on the axes). In most cases, the model used to generate the data provided the best fit for the generated data, as reflected by the darkest square in each column being on the diagonal. However, the fitting procedure could not perfectly distinguish between certain quantities used for generating the value of individual gamble outcomes: $m$ and $u(m)$; $p$ and $w(p)$; and EV, EV with $w(p)$, EU, and SU. B, The same as in $\boldsymbol{A}$ but when AIC values were normalized by the maximum value in each column. Figure 3-1, available at https://doi.org/10.1523/JNEUROSCI.252218.2019.f3-1, shows that our fitting method was able to correctly identify the type of strategy (single- vs combined-attribute) used for evaluating individual gamble outcomes in majority of cases. Figure 3-2, available at https://doi.org/10.1523/JNEUROSCl.2522-18.2019.f3-2, shows that our fitting method can accurately recover model parameters without any systematic bias.

via a simple attentional mechanism. More specifically, attention could be guided by different types of reward information (e.g., reward magnitude, expected value, etc.) to allow for differential weighting of possible outcomes, perhaps via gain modulation. This resulted in a large family of 240 unique models that differed in their assumptions about the strategies used for the evaluation of individual outcomes of a given gamble (eight possible quantities; Fig. $1 C$ ), for sorting and assigning different weights to individual outcomes (six possible quantities), and for combining outcome values to construct the overall value of a complex gamble (5 possible strategies; Fig. 1D; see Materials and Methods). For model comparison, we used the AIC and/or BIC to account for different numbers of parameters in different models.

Considering the complexity of the proposed models, we first tested whether our fitting procedure could identify the underlying mechanisms for the construction of overall reward value and estimate the associated parameters correctly. More specifically, we simulated choice data using the proposed models over a wide range of model parameters for the same complex-gamble task performed by the subjects (see Materials and Methods). We subsequently fit the simulated data with each of these models to compute the goodness-of-fit (in terms of AIC) and to estimate the original model parameters used to simulate the data.

We found that for most cases, the model used to generate the data provided the best fit, indicating that the fitting could be used to identify the mechanism underlying valuation (Fig. 3). We also found that the fitting procedure could not perfectly distinguish between certain strategies used for the evaluation of individual outcomes: $m$ and $u(m) ; p$ and $w(p)$; and EV, EV with $w(p), \mathrm{EU}$, and SU. As a result, we took caution when interpreting the fitting results of subjects' actual data with regard to the exact strategies used to evaluate individual outcomes. 
In addition, we examined the overall accuracy of our method in identification of the strategy used for evaluating individual outcomes. First, we fit choice data generated by a given model with each of the 24 possible models and computed the "average" AIC across all the models with single-attribute evaluation and those with combined-attribute evaluation. This was done separately for sorting based on magnitude, probability, or EV. We found that the average AIC across all types of sorting was smaller for the correct model (Fig. 3-1A-C, available at https://doi.org/ 10.1523/JNEUROSCI.2522-18.2019.f3-1, diagonals have smaller AIC values than off-diagonals). However, the average AIC of the combined-attribute models was only slightly worse than that of the single-attribute models for fitting data generated with singleattribute strategies when sorting based on magnitude (Fig. 3-1 A, available at https://doi.org/10.1523/JNEUROSCI.2522-18.2019. f3-1). This finding was not observed when sorting based on probability or EV (Fig. 3-1 B, C, available at https://doi.org/10.1523/ JNEUROSCI.2522-18.2019.f3-1).

Average AIC values, however, are not very informative about correct model identification since the best model is determined based on the minimum AIC and not the average AIC. Therefore, for a given set of data, we also computed the difference between normalized AIC for the best models (models with minimum AIC) based on single- and combined-attribute evaluation strategies. We found significant differences between the AIC values supporting the correct model (Fig. 3-1 D-F, available at https:// doi.org/10.1523/JNEUROSCI.2522-18.2019.f3-1). Although smaller for data generated with single-attribute evaluation strategies and magnitude sorting, differences in AIC for the best models allowed the correct model to be identified in majority of cases. More specifically, only in 3-15\% of the instances was the type of evaluation strategy incorrectly identified, such as when using data generated with single-attribute evaluation and sorted based on magnitude (Fig. 3-1G-I, available at https://doi.org/10.1523/ JNEUROSCI.2522-18.2019.f3-1). Overall, these analyses demonstrate that the error in identification of the type of evaluation strategy (single- vs combined-attribute evaluation) is relatively small.

Finally, we validated our fitting method by comparing estimated and actual model parameters. We found the overall relative differences between the actual parameters and the parameters estimated by the same model used for generating the data to be very small, which indicates little to no systematic biases in our analysis (Fig. 3-2, available at https://doi.org/10.1523/ JNEUROSCI.2522-18.2019.f3-2). Together, these simulation results support the feasibility of our fitting approach for identifying mechanisms used for the construction of overall reward value in the real data.

\section{Subjects used different quantities to evaluate and sort the} outcomes of complex gambles

We next fit individual subjects' data with our models to identify the strategies used for sorting, evaluating, and weighting of gamble outcomes. We computed and compared the goodness-of-fit across all models based on all combinations of strategies to find the best overall model for each subject. We also examined the quality of model selection using Vuong's test.

We first examined the quantity used by subjects to sort the possible outcomes of complex gambles into best, middle, and worst outcomes to differentially weigh them. We found that $61 \%$ of subjects sorted gamble outcomes based on outcomes' reward magnitudes or probabilities (Fig. 4A). This percentage was larger than the percentage of subjects who used a combination of re- ward information (EV, EV with $w(p)$, EU, or $\mathrm{SU})$ for sorting outcomes $\left(\chi_{(1)}^{2}=5.28, p=0.012\right)$.

We then compared quantity or strategy used by each subject to evaluate individual gamble outcomes. We computed the percentage of subjects who used a given quantity to evaluate individual outcomes, as determined by the model that provided the best fit in terms of the AIC (or BIC, see the last paragraph of this section). We found that $41,25,16$, and $12 \%$ of subjects evaluated individual outcomes based on EU, SU, EV, and EV with $w(p)$, respectively (Fig. $4 B$ ). These results demonstrate that most subjects $(60$ of 64 , corresponding to $94 \%$ of subjects) combined information about reward probability and magnitude to evaluate individual outcomes of complex gambles.

Therefore, although $94 \%$ of subjects combined reward information to evaluate individual gamble outcomes, most subjects used a single piece of reward information when sorting outcomes for weighting. We found similar dissociation between the strategies used for sorting and evaluating individual outcomes based on the mean AIC across all subjects instead of the best model for individual subjects (Fig. 4-1, available at https://doi.org/10. 1523/JNEUROSCI.2522-18.2019.f4-1). This stark difference demonstrates that separate mechanisms were involved in evaluating and combining the values of individual outcomes to construct an overall value for complex gambles.

As mentioned earlier, our method could misidentify singleattribute evaluation strategies with combined-attribute ones in $<\sim 15 \%$ of model instances when sorting based on magnitude. Considering this relatively small error rate and the fact that we ensured that goodness-of-fit for the best model is significantly better than the second best model (using Vuong's test), we estimate that model misidentification could potentially result in mislabeling only a few subjects $(<9)$, which does not change our main conclusion. However, the fitting procedure could misidentify the exact evaluation strategy among combined-attribute strategies (i.e., EV, EV with $w(p)$, EU, and SU), and thus, we used caution in interpreting the exact strategy used for combinedattribute evaluation.

To determine how subjects combined outcome values to form the overall value of a gamble (or equivalently, to directly compare gambles), we then compared five alternative models of outcome weighting (Fig. 1D). This analysis revealed that weighting Strategy 4, which assigned different weights to the three possible outcomes, provided the best fit for a majority $(80 \%)$ of the subjects (Fig. 4C). Moreover, choice behavior of only 5 of 64 subjects $(8 \%)$ were best fit by Strategies 2 and 3 (which ignored the worst outcome), indicating that the majority of subjects (92\%) considered the values of all three possible gamble outcomes when making decisions.

To examine whether the best model for each subject is significantly better than the rest of the models, we performed Vuong's test (Vuong, 1989; see Materials and Methods). We found that 19 subjects ( $\sim 30 \%$ of subject) showed no significant difference between the best model (model with minimum LL value) and the second best model. However, for the majority of subjects (45 subjects equal to $70 \%$ of subjects), we found the same pattern of results as in our original analysis using all data (Fig. $4 D-F)$.

Finally, to further illustrate that our results do not depend on the specific metric for the goodness-of-fit, we repeated our model selection and strategy identification based on the BIC. As shown in Fig. 4-2, available at https://doi.org/10.1523/JNEUROSCI. 2522-18.2019.f4-2 and Fig. 4-3, available at https://doi.org/ 10.1523/JNEUROSCI.2522-18.2019.f4-3, we found qualitatively similar results using BIC as the goodness-of-fit and our main 
A

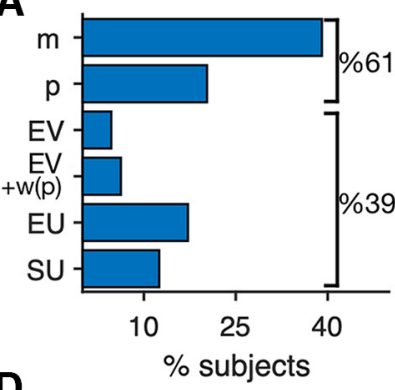

D

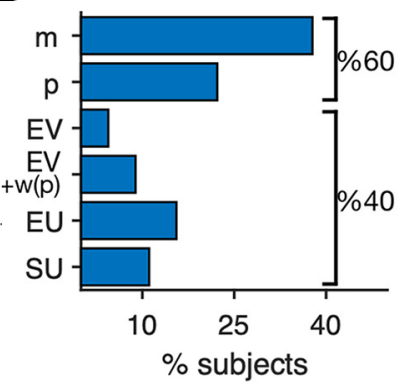

B

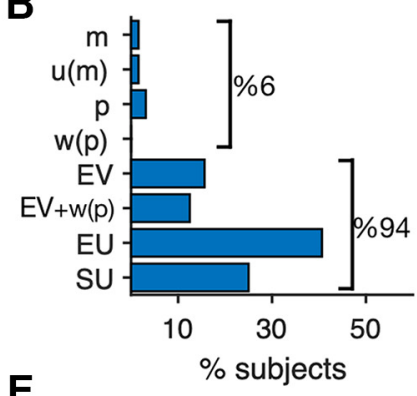

E

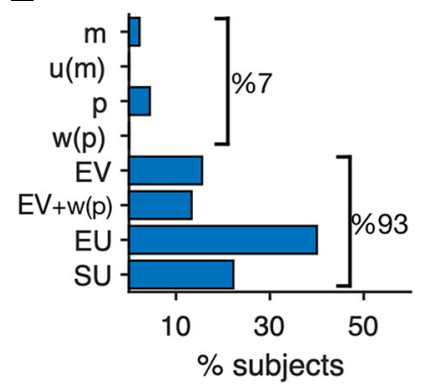

C

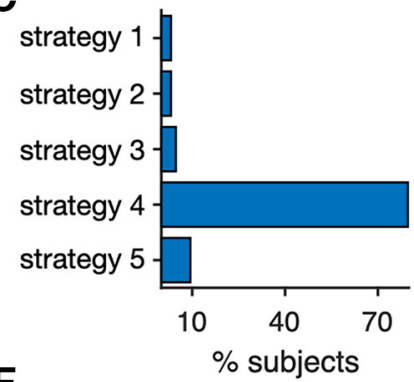

F

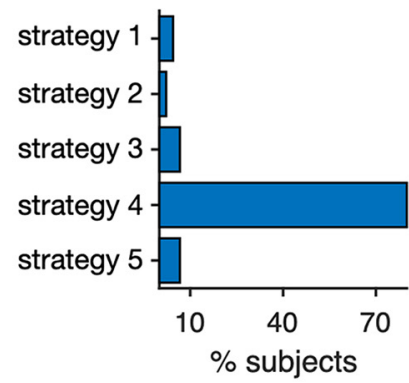

Figure 4. Most subjects sorted and weighted possible outcomes based on reward magnitude or probability (single-attribute sorting) but evaluated each gamble outcome based on a combination of reward attributes (combined-attribute evaluation). $\boldsymbol{A}$, Plot shows the fraction of subjects whose choice was best fit by a given quantity for sorting, and the sum over all single-attribute vs combined-attribute sorting. Overall, most subjects (61\%) used a single reward attribute for sorting gamble outcomes; reward magnitude was the most used quantity (40\%) followed by reward probability (21\%). Note that identical results were obtained from sorting outcomes based on $m$ and $u(m)$, and for $p$ and $w(p)$. $\boldsymbol{B}$, Plot shows the fraction of subjects whose choice was best fit by a given strategy to evaluate individual gamble outcomes. Overall, most subjects (94\%) used a combination of reward attributes for evaluating individual gamble outcomes, with EU being the most used quantity (41\%). C, Plot shows the fraction of subjects whose choice was best fit by a given weighting strategy. Weighting Strategy 4 provided the best fit for the majority (51 of 64 , corresponding to $80 \%$ ) of subjects. $\boldsymbol{D}-\boldsymbol{F}$, Plots are similar to $\boldsymbol{A}-\boldsymbol{C}$ but show the results for subset of subjects for whom the fit based on their best model was significantly different from other models using Vuong's test $(N=45)$. Thirty-eight percent of subjects sorted gamble outcomes based on reward magnitude, whereas $22 \%$ used reward probability for sorting (D). Forty percent of subjects used EU to evaluate individual outcomes in complex gambles $(\boldsymbol{E})$. Strategy 4 provided the best fit for the majority (36 of 45 , corresponding to $80 \%$ ) of subjects $(\boldsymbol{F})$. Figure $4-1$, available at https://doi.org/ 10.1523/JNEUROSCI.2522-18.2019.f4-1, shows qualitatively similar results are obtained when using the average AIC to determine the best overall strategies for sorting, evaluating, and weighting. Figure 4-2, available at https://doi.org/10.1523/JNEUROSCI.2522-18.2019.f4-2, and Figure 4-3, available at https://doi.org/10.1523/JNEUROSCI.2522-18.2019.f4-3, show that similar results are obtained using BIC as the goodness-of-fit measure.

conclusions about the strategies used for sorting, evaluating, and weighting of individual outcomes still hold.

Differential weighting could not be captured by PT

Considering the complexity of models used for fitting, we also examined whether the proposed differential weighting is necessary to capture choice behavior (i.e., our results are not affected by overfitting) beyond what can be explained by changes in the utility and probability weighting functions in PT. In addition, we also tested whether our fitting approach could identify the underlying model parameters without any systematic bias in the presence and absence of differential weighting (an extension of the analyses presented in Fig. 3).

To that end, we generated choice data based on three models that evaluated individual gamble outcomes using subjective value (the most common quantity used for evaluation among our subjects), sorted these outcome values based on magnitude, probability, or expected value, and then combined these outcome values based on Strategy 4 (the most common weighting strategy used among our subjects) using a wide range of weighting parameters similar to those used for simulations presented in Figure 3. We then fit these simulated data with each of the models used to generate the data as well as with the model without differential weighting.

We found that the model with differential weighting was able to fit simulated choice data very well and captured the original model parameters without any bias and with small error in most cases (Fig. 5 and Fig. 5-1, available at https://doi.org/10.1523/ JNEUROSCI.2522-18.2019.f5-1, Fig. 5-2, available at https://
doi.org/10.1523/JNEUROSCI.2522-18.2019.f5-2, Fig. 5-3, available at https://doi.org/10.1523/JNEUROSCI.2522-18.2019.f5$3)$. In contrast, the model without differential weighting was not able to fit the simulated data well and provided systematically biased estimates of risk-preference parameters. More specifically, the estimated $\rho$ and $\gamma$ values based on the model without differential weighting were smaller than the actual values (Fig. 5 and Fig. 5-2, available at https://doi.org/10.1523/JNEUROSCI.252218.2019.f5-2). This indicates that some of the previously observed concavity of the utility function and curvature of the inverse-S-shaped probability weighting function could be due to differential weighting mediated via attentional mechanisms. Together, these results not only validate our approach but also illustrate that prospect theory cannot be used to fit data for which value construction is influenced by differential weighting.

\section{Our model accounts for choice behavior better than} competing models

To compare our model and competing models for valuation of complex gambles, we used four rank-dependent models to fit our experimental data. This includes CPT, TAX, STC, and DFT (see Materials and Methods).

We found that our model can better predict choice behavior for the majority (55\%) of subjects (Fig. 6A). We also compared the ability of our model versus each of the competing models and found that the best competing model (STC) could fit data better for only one-third of subjects (percentage of subjects that were better fit with a competing model: $\mathrm{CPT}=14 \%$; $\mathrm{TAX}=20 \%$; DFT $=12 \%$; STC $=32.8 \%$; Fig. $6 B$ ). Overall, these results dem- 

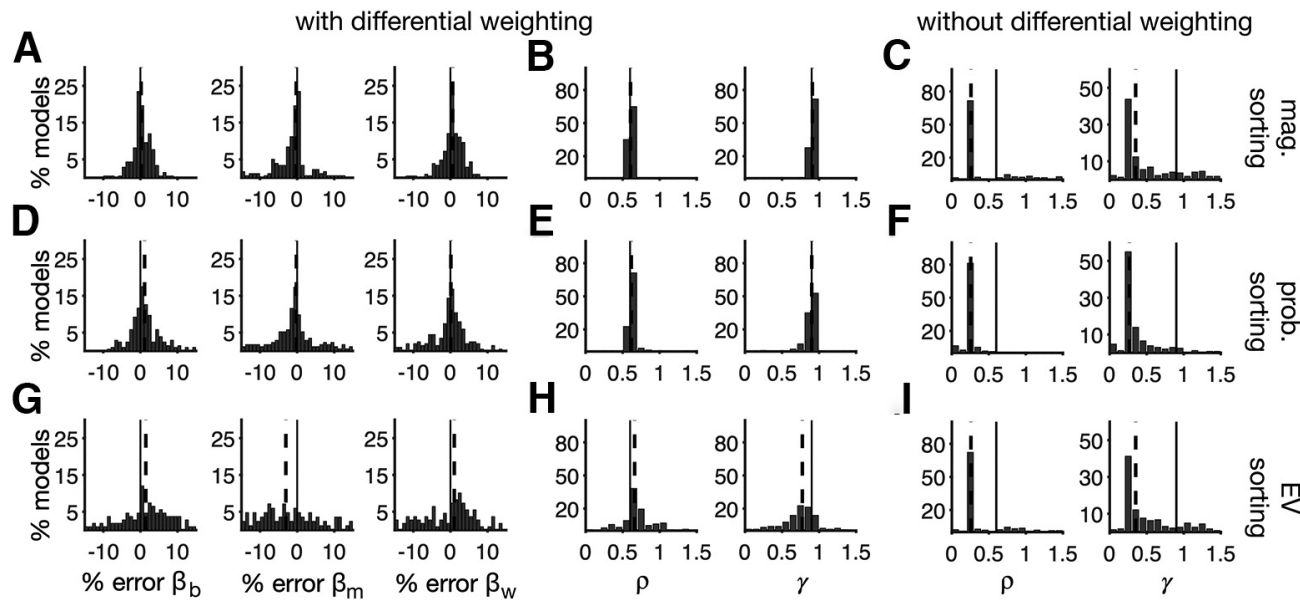

$\mathrm{H}$
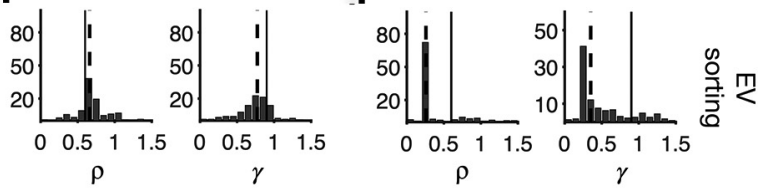

Figure 5. The model with differential weighting can estimate original parameters without a significant bias, whereas the model without differential weighting systematically underestimates $\rho$ and $\gamma$. A, Plots show the distribution of the relative error in the estimation of weights assigned to the best, medium, and worst outcomes (from left to right). For these simulations, the reward values of individual gambles were computed using nonlinear utility and probability weighting functions on the average risk-preference in our subjects ( $\rho=0.6$ and $\gamma=0.9$ ), and outcomes were sorted based on reward magnitude. $\boldsymbol{B}$, The distribution of estimated values for $\rho$ and $\gamma$ across all models. Overall, the estimated parameters were centered around the actual values. $\boldsymbol{C}$, The distribution of estimated values for $\rho$ and $\gamma$ when choice data were fit with a model without differential weighting. The estimated parameters were significantly smaller than the actual values. $\boldsymbol{D}-\boldsymbol{F}$, The same as $\boldsymbol{A}$-C but for sorting based on reward probability. $\mathbf{G}-\boldsymbol{I}$, The same as $\boldsymbol{A}$-C but for sorting based on EV. There was a small systematic bias in estimated parameters when the choice data were generated with sorting based on EV. Nevertheless, this bias does not affect our results because most of our subjects sorted outcomes based on reward magnitude or probability (and not EV). Figure 5-1, available at https://doi.org/10.1523/JNEUROSCI.2522-18.2019.f5-1, shows that the model without differential weighting cannot fit choice data generated with differential weighting and nonlinear utility and probability weighting functions. Figure 5-2, available at https://doi.org/10.1523/JNEUROSCI.2522-18.2019.f5-2, and Figure 5-3, available at https://doi.org/10.1523/ JNEUROSCI.2522-18.2019.f5-3, show similar results when using data generated with linear utility and probability weighting functions.

onstrate that our plausible yet simpler model can outperform more complex models in capturing choice behavior. Importantly, our model distinctly allows for the evaluation of individual outcomes and their combination to rely on different pieces of information (e.g., evaluation based on expected value but differential weighting based on magnitude). Therefore, the superiority of our model in capturing individual subjects' choice behavior could indicate that individual variability in evaluating complex gambles could arise from differences in the type of reward information that guides attention.

Subjects assigned larger weights to the best and worst outcomes in terms of reward magnitude and probability

Having established that most subjects used differential weighting to combine reward values of possible outcomes, we then examined the weights assigned to the three outcomes within a gamble. For subjects who sorted outcomes based on a single reward attribute, we observed significant differences in weight assignments (Fig. 7A-D). More specifically, the weight assigned to the best (largest magnitude or probability) outcome was significantly greater than that of the middle outcome $\left[\beta_{\mathrm{b}}-\beta_{\mathrm{m}}=0.13 \pm 0.27\right.$ (mean $\pm \mathrm{SD}$ ), two-sided sign test, $p=0.02, d=0.44$; Fig. $7 C]$. The worst outcome also had a significantly greater weight compared with the middle outcome $\left[\beta_{\mathrm{w}}-\beta_{\mathrm{m}}=0.09 \pm 0.37\right.$ (mean $\pm \mathrm{SD}$ ), two-sided sign test, $p=0.0005, d=0.23$; Fig. $7 D]$. There was no significant difference between the weights for the best and worst outcomes (two-sided sign test, $p=0.61, d=0.11$ ).

These results illustrate that the most important outcomes (best and worst based on a given subject's sorting) were assigned larger weights for the construction of overall reward value. In contrast, for subjects who sorted outcomes based on a combination of reward information (EV, EV with $w(p), \mathrm{EU}$, or $\mathrm{SU})$, there were no significant weight differences between the best and middle outcomes $\left[\beta_{\mathrm{b}}-\beta_{\mathrm{m}}=0.06 \pm 0.30\right.$ (mean $\pm \mathrm{SD}$ ), two-sided sign test, $p=0.7, d=0.17$; Fig. $7 E-G]$ or between the worst and middle outcomes $\left[\beta_{\mathrm{w}}-\beta_{\mathrm{m}}=-0.08 \pm 0.45(\right.$ mean $\pm \mathrm{SD})$, two-sided sign test, $p=0.98, d=0.19$; Fig. $7 H]$. These results indicate that differential weighting of gamble outcomes was consistent mainly among subjects who sorted outcomes based on a single piece of reward information.

To address the robustness and consistency of our method and estimated model parameters, we also measured the variability in the estimation of model parameters. More specifically, we used the best model for a given subject to fit $90 \%$ of choice data (randomly sampled) from that subject and estimated all model parameters. We then repeated this procedure 100 times to calculate the distributions of estimated parameters for each subject. Using the distribution of each model parameter, we then computed the 

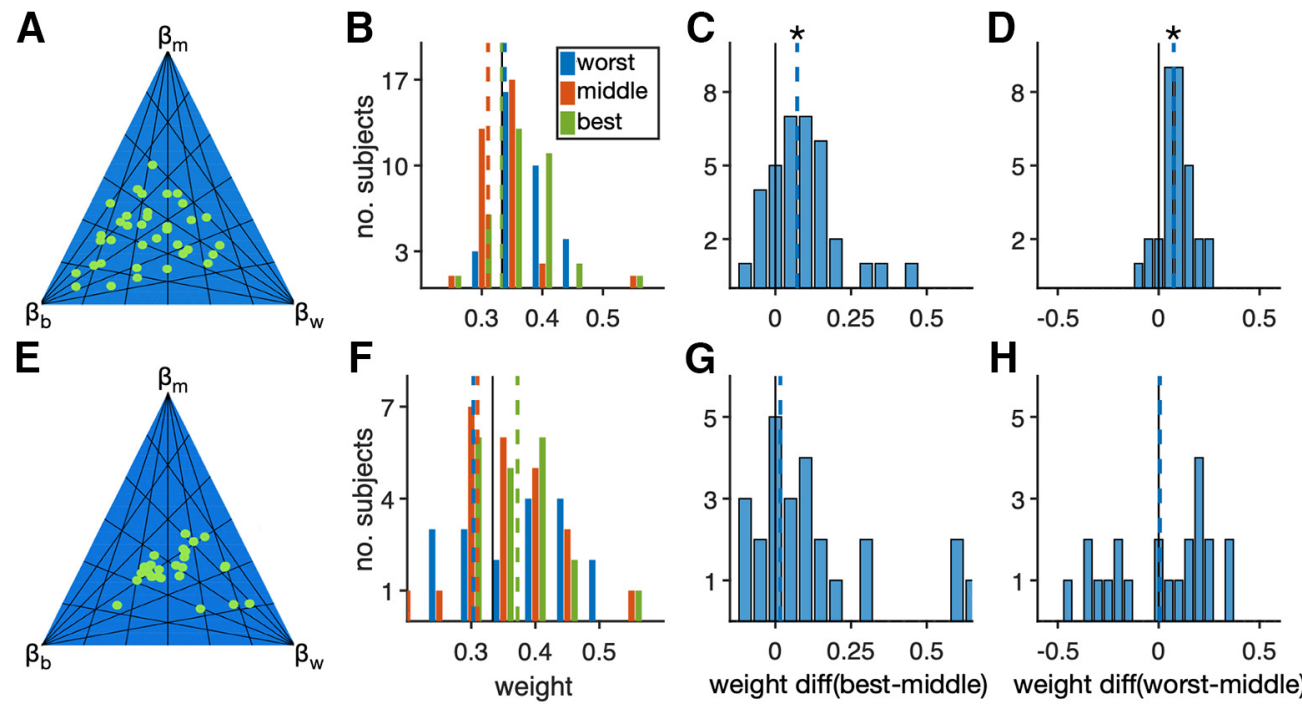

Figure 7. Differential weighting was consistent among subjects who sorted outcomes based on either reward magnitude or probability. $A$, The ternary plot shows the values of differential weighting for subjects who used a single gamble attribute to sort possible outcomes $(N=39)$. Each dot represents a set of weights as indicated by the distance of the dot from the three corners. $\boldsymbol{B}$, Plot shows the distribution of absolute weight values assigned to each outcome within a three-outcome gamble. Dashed and solid lines represent median and 0.33 , respectively. The median weights assigned to the best, middle, and worst outcomes (as determined by either reward magnitude or probability) were $0.33,0.31$, and 0.34 , respectively. $C$, Plot shows the distribution of relative weight differences between the best and middle outcomes. The relative weights were computed by normalizing the two weights by their sum. Blue dashed and solid gray lines represent the median and 0 , respectively, and the asterisk indicates that the median difference was significantly different from zero (two-sided sign test, $p<0.05$ ). $D$, Plot shows the distribution of relative weight differences between the worst and middle outcomes. Conventions are the same as in $\boldsymbol{C} . \boldsymbol{E}-\boldsymbol{H}$, Plots are similar to $\boldsymbol{A}-\boldsymbol{D}$ but show the results for subjects who used a combination of reward information (EV, EV with $w(\boldsymbol{p})$, EU, or SU) for sorting and assigning weights $(N=25)$. Figure 7-1, available at https://doi.org/10.1523/JNEUROSCI.2522-18.2019.f7-1, shows that our method can estimate weighting parameters consistently. Figure 7-2, available at https://doi.org/10.1523/JNEUROSCI.2522-18.2019.f7-2, shows that observed differential weighting was not driven by specific color-reward assignments.

coefficient of variation (CV; equal to the SD of the distribution divided by its mean), as a standardized measure of dispersion in the estimated model parameters. Small values of CV indicate consistency or robustness of estimated parameters. To provide a baseline, we also computed CV for model parameters in the simple-gamble task using the same procedure described above.

Overall, we found relatively small CV values for the estimated weighting parameters of all reward outcomes (median $\mathrm{CV}=$ $0.21,0.17$, and 0.25 for the best, middle, and worst outcomes, respectively; Fig. 7-1, available at https://doi.org/10.1523/ JNEUROSCI.2522-18.2019.f7-1). As a baseline for comparison, the median of $\mathrm{CV}$ of the estimated model parameters $(\rho, \gamma$ and $\sigma)$ across subjects in the simple-gamble task was 0.16 . These results illustrate that our method can estimate model parameters consistently, and thus, our method can be used to make reliable inferences about differential weighting of possible outcomes.

In our study, reward magnitude was represented by specific combinations of colors for different subjects. Therefore, we also examined that our observations were not driven by certain combinations of color-reward assignments (e.g., red for the largest reward could be more effective than green); that is, there was no systematic color bias. We categorized subjects into six possible groups based on their color-reward assignments and examined differential weighting for each group (Fig. 7-2, available at https:// doi.org/10.1523/JNEUROSCI.2522-18.2019.f7-2). This analysis did not reveal any evidence for systematic differences between the groups, indicating that differential weighting of possible outcomes was unlikely because of specific color-reward assignments.

Larger weighting of the best and worst outcomes was not driven by information seeking

In designing complex gambles, we only ensured that the subjective utilities of each gamble pairs were close in value for each subject. Therefore, it is possible that the distribution of the probability of middle outcomes across all trials was less variable (or disperse) than those of the best and worst outcomes. A larger dispersion of the distributions for the best and worst outcome probabilities may result in these outcomes to be perceived as more informative and thus, weighted more strongly. To exclude such an explanation for our observation, we computed the distributions of reward probability for the best, middle, and worst outcomes (when sorting based on magnitude) for each subject and calculated SD (as a measure of dispersion) of these distributions across all subjects.

As shown in Figure 8, we did not find any significant difference between the medians of standard deviations of outcome probabilities for the best, medium, and worst outcomes. Therefore, we did not find any evidence for the dispersion of outcome probabilities (and thus the informativeness of outcomes) to underlie the observed overweighting of the best and worst outcomes.

Differential weighting could enable subjects to more easily and quickly choose between complex gambles

To address possible advantages of differential weighting, we examined the influence of this mechanism on overall risk preference and whether it allowed subjects to make decisions more easily. Critically, we designed the complex-gamble task such that the pair of gambles presented on each trial have similar SU (using a wide range of reward probabilities) to detect additional mechanisms involved in the construction of reward value (see Materials and Methods). This feature also made decision making more difficult in the complex-gamble task.

To quantify the influence of differential weighting on overall risk preference, we computed the relative change in the value of each gamble after the inclusion of differential weighting based on estimated weights for each subject (for all gambles used in the complex-gamble task). We found that when subjects used reward magnitude for sorting, assigning a larger weight to the best outcome (i.e., outcome with the largest magnitude) resulted in an increase in the value of gambles, thereby increasing risk-seeking 
A

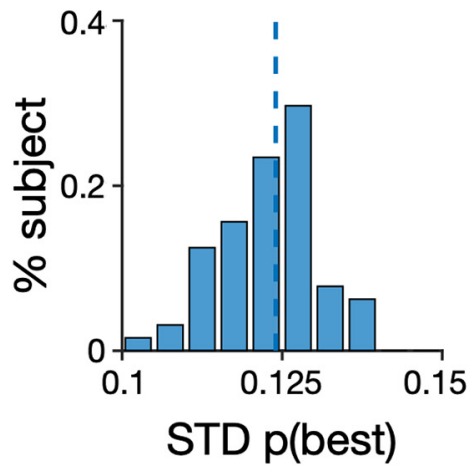

B

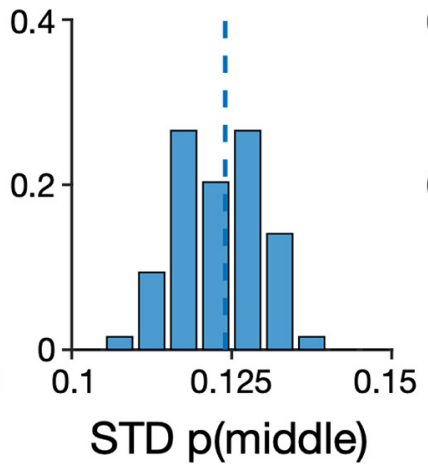

C

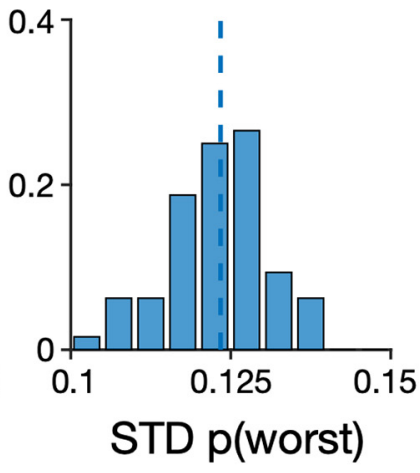

Figure 8. Distributions of outcome probabilities for the best, medium, and worst outcomes had similar dispersion. Plots show the distributions of SD for the outcome probabilities for best (A), middle $(\boldsymbol{B})$, and worst $(\boldsymbol{C})$ outcomes (based on magnitude) across all subjects. Dashed lines show medians of the distributions. There was no significant difference between the medians of the distributions of SD for the three outcomes (two-sided sign test; best vs middle: $p=1.0, d=0.01$; best vs worst: $p=0.38, d=0.03$; middle vs worst: $p=0.38, d=0.01$ ).

A
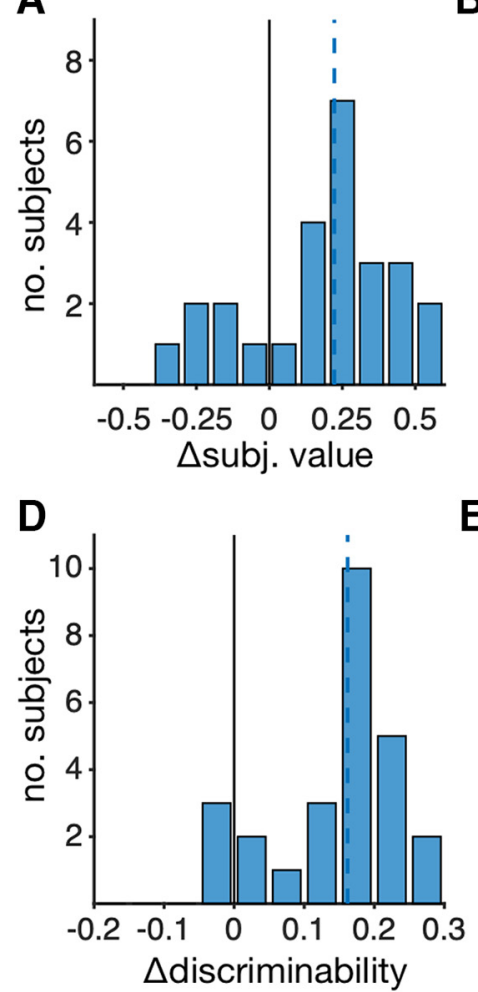

B

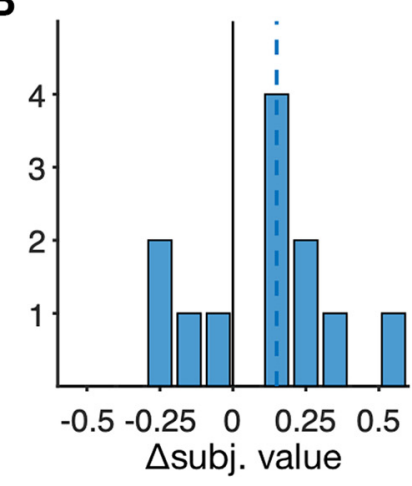

E

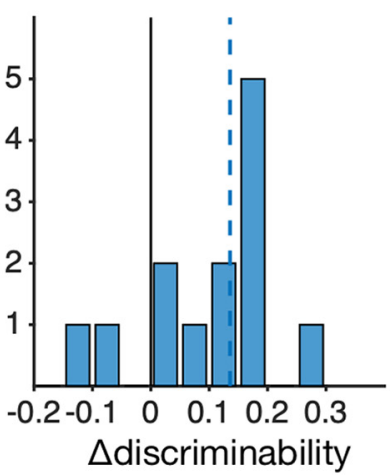

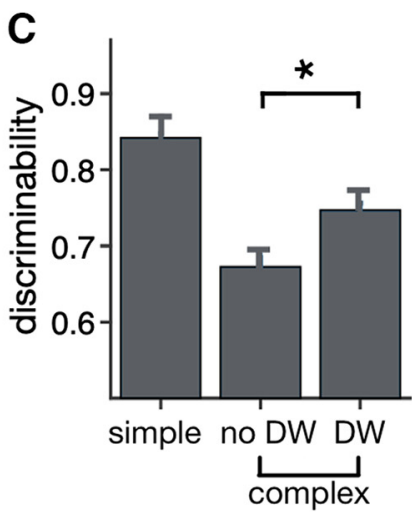

$\mathbf{F}$

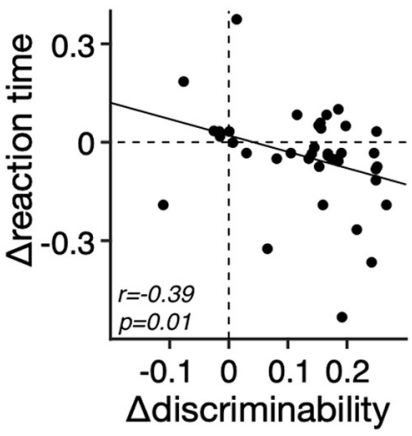

Figure 9. Differential weighting could enable subjects to make decisions more easily and quickly. $\boldsymbol{A}, \boldsymbol{B}$, Influence of differential weighting on risk preferences. Plots show the distributions of changes in subjective value of a three-outcome gamble after including differential weighting based on estimated weights for individual subjects who used reward magnitude $(\boldsymbol{A})$ or probability $(\boldsymbol{B})$ for sorting. Dashed lines represent the median, which were equal to 0.19 and 0.16 in $\boldsymbol{A}$ and $\boldsymbol{B}$, respectively, and were significantly different from 0 (two-sided sign test; $p=7.8 \times 10^{-3}$, $\left.6.2 \times 10^{-3} ; d=0.88,0.92\right)$. C, Plot shows the discriminability of subjects for the simple-gamble (simple) task as well as the complex-gamble task based on the models with and without differential weighting (DW). The inclusion of differential weighting significantly increased discriminability in the complex-gamble task, as shown by the asterisk (tw0-sided sign test, $p=1.4 \times 10^{-10}, d=$ 0.51). $\boldsymbol{D}, \boldsymbol{E}$, Influence of differential weighting on discriminability. Plots show the distributions of changes in discriminability between pairs of three-outcome gambles after including differential weighting based on estimated weights for individual subjects who used reward magnitude $(\boldsymbol{D})$ or probability $(\boldsymbol{E})$ for sorting, both of which are significantly different from 0 (median $=0.15,0.14$; two-sided sign test; $\left.p=1.4 \times 10^{-3}, 2.6 \times 10^{-4} ; d=0.96,1.02\right)$. Other conventions are similar to those in $\boldsymbol{A}$ and $\boldsymbol{B}$. $\boldsymbol{F}$, Plotted is the change in median reaction time between the simple- and complex-gamble tasks versus the corresponding change in discriminability due to differential weighting for individual subjects. Overall, a decrease in reaction time from the simple-gamble to complex-gamble task was correlated with an increased discriminability between the two tasks. Figure 9-1, available at https://doi.org/10.1523/JNEUROSCI.2522-18.2019.f9-1, shows changes in subjective value of a three-outcome gamble after including differential weighting with a given set of weights, reward probabilities, reward magnitudes, and using magnitude for sorting.

behavior (Fig. 9A). Similar but weaker changes in subjective values were observed in the case of sorting based on reward probability (Fig. 9B).

To better understand how risk preference is influenced by differential weighting, we also computed the relative change in the value of each gamble after the inclusion of differential weight- ing using a wide range of weights and reward probabilities when sorting based on magnitude (Fig. 9-1, available at https://doi.org/ 10.1523/JNEUROSCI.2522-18.2019.f9-1). We found that the change in overall value depended on which outcome was assigned the largest weight. More specifically, the overall value of a threeoutcome gamble always increased if the outcome with the highest 
expected value (or subjective utility) was assigned the largest weight, resulting in more risk-seeking behavior. In contrast, the overall value decreased if the outcome with the lowest expected value was assigned the largest weight, resulting in more riskaversive behavior.

To quantify how easily a given subject could distinguish between pairs of gambles, we defined discriminability based on the subjective values of gambles estimated for that subject (see Materials and Methods). We computed and compared discriminability for each subject in the complex-gamble task as well as in the simple-gamble task using the best subject-specific models with and without differential weighting, respectively. We found the effect of differential weighting on discriminability to be more complex than that on the overall gamble value, but overall, differential weighting increased discriminability for most weight values (Fig. 9D,E). As expected, discriminability was smaller across all subjects in the complex-gamble task compared with the simple-gamble task because of differences in task design (Fig. 9 C). The inclusion of differential weighting, however, significantly increased discriminability across all subjects during the complex-gamble task (two-sided sign test, $p=1.4 \times 10^{-10}, d=$ 0.51 ). In other words, subjects who used differential weighting could more easily discriminate and thus, choose between gambles.

To test whether this increase in discriminability also allowed for faster decision making, we examined the correlation between the change in the average reaction time between the simple- and complex-gamble tasks and the corresponding change in discriminability because of differential weighting within individual subjects. We found a significant negative correlation between the change in reaction time and the change in discriminability for subjects who sorted outcomes based on reward probability or magnitude (Pearson correlation: $r=-0.32, p=0.035$; Spearman correlation, $r=-0.39, p=0.017$; Fig. $9 F)$. A similar result was found when considering all subjects (Pearson correlation: $r=-0.34, p=0.006$; Spearman correlation, $r=-0.38, p=$ 0.002 ). This indicates that subjects became relatively faster in the complex-gamble task depending on the extent to which they used differential weighting for discrimination between gambles. Together, these results suggest that differential weighting enabled subjects to more easily and quickly select between three-outcome gambles.

\section{Discussion}

By comparing choice between simple and three-outcome gambles within subjects, we examined how valuation and decisionmaking between more complex gambles are influenced by attentional mechanisms. We found that choice between simple gambles was consistent with prospect theory since evaluation based on subjective utility provided the best fit for our data. When evaluating three-outcome gambles, subjects also combined reward probability and magnitude to assign a value to each gamble outcome, but at the same time, most subjects differentially weighted possible outcomes based on either reward magnitude or probability. These results point to a novel dissociation between how reward information of complex gambles is processed: valuation of each outcome is based on a combination of reward information, whereas weighting of possible outcomes mainly relies on a single piece of reward information. This flexible weighting of possible outcomes, in turn, allowed for a more dynamic construction of reward value and enabled easier and faster decision making, especially for difficult choices between options with similar objective or subjective values. Together, our study reveals a plausible, salience-driven mechanism underlying valuation of complex gambles.

Currently, there are a number of sophisticated models for valuation and choice between complex gambles. Most of these models rely on a rank-dependent mechanism for processing alternative outcomes. Although our model does not use cumulative weighting function, its weighting mechanism makes it resemble the STC model. Unlike our model, however, STC and other competing models examined here require complex computations, and it is unclear how these computations can be instantiated in the brain. In addition, none of these competing models have been used to fit choice data from individual subjects, and thus, there is no evidence that they can capture individual variability. Although choice behavior of some subjects is better captured by more complex models, our heuristic model provides a better and more plausible fit for the majority of subjects.

Given our limited processing resources, exhaustively weighting and summating all possible outcomes to evaluate an option is not feasible unless we can simplify valuation and decisionmaking processes using some form of heuristics (Gigerenzer and Goldstein, 1996; Brandstätter et al., 2006; Gigerenzer and Gaissmaier, 2011). In many cases, information must somehow be prioritized to avoid cognitive overload. Such prioritization has been assumed to be performed mainly via attentional mechanisms (Treisman and Gelade, 1980; Klein, 1988; McLeod et al., 1988; Wolfe and Horowitz, 2004; Watson and Kunar, 2010; Russell and Kunar, 2012). It has been shown that both bottom-up and top-down attention can influence processing and integration of reward information and ultimately choice behavior (Krajbich et al., 2010; Tsetsos et al., 2012; Kunar et al., 2017). By selectively processing certain outcomes within complex risky options, the decision maker can reduce computational demands required for evaluation of such options.

In the case of multi-attribute options, decision making can be very difficult because it requires weighting the pros and cons of options that differ across multiple, sometimes incommensurate, dimensions (Fellows, 2006). Therefore, various heuristics have been proposed for reducing the computational demands and complexity of valuation and decision processes in such cases (even in the absence of risk). This includes differential weighting of different dimensions, limiting the amount of information, and reducing the number of alternatives to be considered (Payne et al., 1993; Gigerenzer and Goldstein, 1996; Gigerenzer and Todd, 1999). Although we assumed that each gamble is assigned an overall subjective value, decision making in our model can also be interpreted as weighted comparisons across individual outcomes based on reward probability or magnitude; that is, the subjects could directly choose between gambles by comparing values for similar individual outcomes and combine such comparisons across all possible outcomes (Tversky, 1969, 1972). This further illustrates how attention can modulate choice between risky options by differentially weighting various comparisons across possible attributes or outcomes.

Critically, we observed a dissociation between what drives the evaluation of individual outcomes (i.e., a combination of reward information) and what drives the weighting process (i.e., a single piece of reward information), which indicates that selective processing of reward information may not rely on a combination of probability and magnitude. The sorting of outcomes based on a single reward attribute can be seen as a more general case of the elimination by aspect theory (Tversky, 1972). One possible mechanism for such processing is selective attention (Busemeyer and Townsend, 1993; Roe et al., 2001; Shimojo et al., 2003; 
Hayden et al., 2008; Ludvig et al., 2014). In our task, the most relevant form of attention is feature-based attention, which could selectively enhance the representation of certain visual attributes (e.g., color or size) at the expense of the others (Carrasco, 2011). Given the visual presentation of gambles in our experiment, subjects could attend to certain "learned" reward features (color and size) within the gamble and thus, weigh outcomes by their reward salience. Interestingly, we found that subjects assigned larger weights to both the best and worst outcomes, indicating that extreme outcomes were most salient. A plausible neural mechanism for this differential weighting could be biased competition associated with feature-based attention (Reynolds et al., 1999; Kastner and Ungerleider, 2001; Beck and Kastner, 2005) or competition at multiple levels of value representation (Jocham et al., 2012; Hunt et al., 2014). The increased weighting of the best outcome in terms of magnitude is consistent with observed increases in attention toward larger rewards (Della Libera and Chelazzi, 2006; Raymond and O'Brien, 2009) and results in riskseeking behavior. In contrast, larger weighting of the worst outcome can contribute to risk-aversion. Together, our results suggest that attentional processes could contribute to differential weighting of reward outcomes by their salience, which simplifies valuation and ultimately results in flexible adjustments of risk attitudes.

The observed dissociation between the types of reward information used for evaluation of individual outcomes and for weighting of alternative outcomes suggests separate mechanisms through which reward influences choice and the selective processing of information (Soltani et al., 2016; Rakhshan et al., 2018) and highlights the importance of attention for adaptive choice under risk at the expense of optimality (Farashahi et al., 2017a,b). More specifically, although each possible outcome should be evaluated based on a strategy that combines different pieces of reward information (and thus could be optimal), differential weighting based on a single quantity (e.g., reward magnitude or probability) can enable flexibility depending on the state of the decision maker. For example, when hungry, the decision maker could attend more to cues that represent the amount of food, or reward. This could result in more risk-seeking behavior but also faster and easier decision making, both of which are crucial for survival. Finally, the success of our model in capturing individual subjects' choice behavior indicates that individual variability in evaluating complex gambles could arise from differences in the type of reward information that guides attention.

Therefore, differential weighting of outcomes provides a plausible mechanism for flexible risk attitudes (Huber et al., 1982; Lattimore et al., 1992; Hey and Orme, 1994; Stewart et al., 2003; Bruhin et al., 2010; Ludvig et al., 2013; Rigoli et al., 2016; Fujimoto and Takahashi, 2016) and results in a tradeoff between optimality and flexibility. We have recently shown that a simpler version of our model can account for monkey's choice behavior during choice between simple gambles, providing further evidence for differential weighting (Farashahi et al., 2018). We speculate that observed deviations from normative theories of choice could be due to such weighting mechanisms, reflecting the flexibility required for decision making in dynamic environments (Payne et al., 1988). Together, our results shed light on possible neural mechanisms of choice under risk in naturalistic settings, and moreover, highlight the role of attention in the flexible construction of reward value for complex gambles.

We assert that although our study does not include neuronal measurements, our extensive model fitting and data analyses provide a simple but plausible mechanism for how value of com- plex gambles are constructed, or equivalently, how these gambles are compared. The main mechanism proposed here (i.e., differential weighting of individual gamble outcomes) is simple enough that can be easily implemented via attentional mechanisms. Specifically, attention can be guided to certain gamble outcomes based on a single attribute and subsequently change (perhaps via gain modulation) the influence of those outcomes on the overall value or choice between a pair of gambles. Our novel finding about the dissociation between how reward information is processed when evaluating complex gambles can be tested in future experiments using neuronal recording.

\section{References}

Abdellaoui M (2000) Parameter-free elicitation of utility and probability weighting functions. Manage Sci 46:1497-1512.

Beck DM, Kastner S (2005) Stimulus context modulates competition in human extrastriate cortex. Nat Neurosci 8:1110-1116.

Birnbaum MH (2008) New paradoxes of risky decision making. Psychol Rev 115:463-501.

Birnbaum MH, Navarrete JB (1998) Testing descriptive utility theories: violations of stochastic dominance and cumulative independence. J Risk Uncertain 17:49-78.

Bordalo P, Gennaioli N, Shleifer A (2012) Salience theory of choice under risk. Q J Econ 127:1243-1285.

Bordalo P, Gennaioli N, Shleifer A (2013) Salience and consumer choice. J Polit Econ 121:803-843.

Brandstätter E, Gigerenzer G, Hertwig R (2006) The priority heuristic: making choices without trade-offs. Psychol Rev 113:409-432.

Bruhin A, Fehr-Duda H, Epper TF (2010) Risk and rationality: uncovering heterogeneity in probability distortion. Econometrica 78:1375-1412.

Busemeyer JR, Townsend JT (1993) Decision field theory: a dynamiccognitive approach to decision making in an uncertain environment. Psychol Rev 100:432-459.

Carrasco M (2011) Visual attention: the past 25 years. Vis Res 51:14841525

Della Libera C, Chelazzi L (2006) Visual selective attention and the effects of monetary rewards. Psychol Sci 17:222-227.

Farashahi S, Donahue CH, Khorsand P, Seo H, Lee D, Soltani A (2017a) Metaplasticity as a neural substrate for adaptive learning and choice under uncertainty. Neuron 94:401-414.e6.

Farashahi S, Rowe K, Aslami Z, Lee D, Soltani A (2017b) Feature-based learning improves adaptability without compromising precision. Nat Commun 8:1768.

Farashahi S, Azab H, Hayden B, Soltani A (2018) On the flexibility of basic risk attitudes in monkeys. J Neurosci 38:4383-4398.

Fellows LK (2006) Deciding how to decide: ventromedial frontal lobe damage affects information acquisition in multi-attribute decision making. Brain 129:944-952.

Fujimoto A, Takahashi H (2016) Flexible modulation of risk attitude during decision-making under quota. Neuroimage 139:304-312.

Gigerenzer G, Gaissmaier W (2011) Heuristic decision making. Annu Rev Psychol 62:451-482.

Gigerenzer G, Goldstein DG (1996) Reasoning the fast and frugal way: models of bounded rationality. Psychol Rev 103:650-669.

Gigerenzer G, Todd PM (1999) Simple heuristics that make us smart. New York: Oxford UP.

Glöckner A, Pachur T (2012) Cognitive models of risky choice: parameter stability and predictive accuracy of prospect theory. Cognition 123:21-32.

Gonzalez R, Wu G (1999) On the shape of the probability weighting function. Cogn Psychol 38:129-166.

Hayden BY, Heilbronner SR, Nair AC, Platt ML (2008) Cognitive influences on risk-seeking by rhesus macaques. Judgm Decis Mak 3:389-395.

Hey J, Orme C (1994) Investigating generalizations of expected utility theory using experimental data. Econometrica 62:1291-1326.

Huber J, Payne JW, Puto C (1982) Adding asymmetrically dominated alternatives: violations of regularity and the similarity hypothesis. J Consum Res 9:90-98.

Hunt LT, Dolan RJ, Behrens TE (2014) Hierarchical competitions subserving multi-attribute choice. Nat Neurosci 17:1613-1622. 
Jocham G, Hunt LT, Near J, Behrens TE (2012) A mechanism for valueguided choice based on the excitation-inhibition balance in prefrontal cortex. Nat Neurosci 15:960-961.

Kahneman D, Tversky A (1979) Prospect theory: an analysis of decision under risk. Econometrica 47:263-291.

Kastner S, Ungerleider LG (2001) The neural basis of biased competition in human visual cortex. Neuropsychologia 39:1263-1276.

Klein R (1988) Inhibitory tagging system facilitates visual search. Nature 334:430-431.

Krajbich I, Armel C, Rangel A (2010) Visual fixations and the computation and comparison of value in simple choice. Nat Neurosci 13:1292-1298.

Kunar MA, Watson DG, Tsetsos K, Chater N (2017) The influence of attention on value integration. Atten Percept Psychophys 79:1615-1627.

Lattimore PK, Baker JR, Witte AD (1992) The influence of probability on risky choice. J Econ Behav Organ 17:377-400.

Ludvig EA, Madan CR, Spetch ML (2013) Extreme outcomes sway risky decisions from experience. J Behav Decis Mak 27:146-156.

Ludvig EA, Madan CR, Pisklak JM, Spetch ML (2014) Reward context determines risky choice in pigeons and humans. Biol Lett 10:20140451.

McLeod P, Driver J, Crisp J (1988) Visual-search for a conjunction of movement and form is parallel. Nature 332:154-155.

Payne JW, Bettman JR, Johnson EJ (1988) Adaptive strategy selection in decision making. J Exp Psychol Learn Mem Cogn 14:534-552.

Payne JW, Bettman JR, Johnson EJ (1993) The adaptive decision maker. New York: Cambridge UP.

Prelec D (1998) The probability weighting function. Econometrica 66: 497-527.

Rakhshan M, Lee V, Chu E, Harris L, Laiks L, Khorsand P, Soltani A (2018) Influence of expected reward on perceptual decision making. bioRxiv 506790.

Raymond JE, O’Brien JL (2009) Selective visual attention and motivation: the consequences of value learning in an attentional blink task. Psychol Sci 20:981-988.

Reynolds JH, Chelazzi L, Desimone R (1999) Competitive mechanisms subserve attention in macaque areas V2 and V4. J Neurosci 19:1736-1753.
Rigoli F, Rutledge RB, Dayan P, Dolan RJ (2016) The influence of contextual reward statistics on risk preference. Neuroimage 128:74-84.

Roe RM, Busemeyer JR, Townsend JT (2001) Multialternative decision field theory: a dynamic connectionist model of decision making. Psychol Rev 108:370-392.

Russell N, Kunar MA (2012) Colour and spatial cueing in low prevalence visual search. Q J Exp Psychol (Hove) 65:1327-1344.

Shimojo S, Simion C, Shimojo E, Scheier C (2003) Gaze bias both reflects and influences preference. Nat Neurosci 6:1317-1322.

Soltani A, Khorsand P, Guo C, Farashahi S, Liu J (2016) Neural substrates of cognitive biases during probabilistic inference. Nat Commun 7:11393.

Stewart N, Chater N, Stott HP, Reimers S (2003) Prospect relativity: how choice options influence decision under risk. J Exp Psychol Gen 132: 23-46.

Strait CE, Blanchard TC, Hayden BY (2014) Reward value comparison via mutual inhibition in ventromedial prefrontal cortex. Neuron 82: 1357-1366.

Treisman AM, Gelade G (1980) Feature-integration theory of attention. Cogn Psychol 12:97-136.

Tsetsos K, Chater N, Usher M (2012) Salience driven value integration explains decision biases and preference reversal. Proc Natl Acad Sci U S A 109:9659-9664.

Tversky A (1969) Intransitivity of preferences. Psychol Rev 76:31-48.

Tversky A (1972) Elimination by aspects: a theory of choice. Psychol Rev 79:281-299.

Tversky A, Kahneman D (1992) Advances in prospect theory: cumulative representation of uncertainty. J Risk Uncertain 5:297-323.

Vuong QH (1989) Likelihood ratio tests for model selection and non-nested hypotheses. Econometrica 307-333.

Watson DG, Kunar MA (2010) Visual marking and change blindness: moving occluders and transient masks neutralize shape changes to ignored objects. J Exp Psychol Hum Percept Perform 36:1391-1405.

Wolfe JM, Horowitz TS (2004) What attributes guide the deployment of visual attention and how do they do it? Nat Rev Neurosci 5:495-501.

Wu G, Gonzalez R (1996) Curvature of the probability weighting function. Manage Sci 42:1676-1690. 